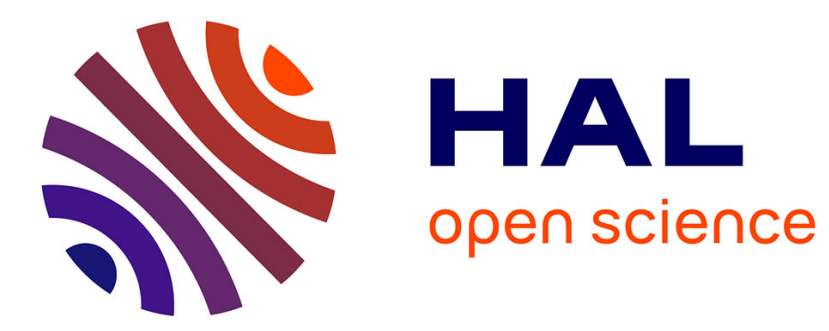

\title{
Topological sensitivity analysis with respect to a small idealized bolt
}

\author{
Lalaina Rakotondrainibe, Grégoire Allaire, Patrick Orval
}

\section{To cite this version:}

Lalaina Rakotondrainibe, Grégoire Allaire, Patrick Orval. Topological sensitivity analysis with respect to a small idealized bolt. Engineering Computations, In press. hal-03157844v2

\section{HAL Id: hal-03157844 \\ https://hal.science/hal-03157844v2}

Submitted on 10 Jun 2021

HAL is a multi-disciplinary open access archive for the deposit and dissemination of scientific research documents, whether they are published or not. The documents may come from teaching and research institutions in France or abroad, or from public or private research centers.
L'archive ouverte pluridisciplinaire HAL, est destinée au dépôt et à la diffusion de documents scientifiques de niveau recherche, publiés ou non, émanant des établissements d'enseignement et de recherche français ou étrangers, des laboratoires publics ou privés. 


\title{
Topological sensitivity analysis with respect to a small idealized bolt
}

\author{
L. Rakotondrainibe G. Allaire P. Orval \\ Technocentre RENAULT, 1 Avenue du Golf, 78084 Guyancourt cedex, France, \\ rakotondrainibe.anja@gmail.com, patrick.orval@renault.com \\ Centre de Mathématiques Appliquées, Ecole Polytechnique, Route de Saclay, 91128 Palaiseau, France, \\ gregoire.allaire@polytechnique.fr
}

June 10, 2021

\begin{abstract}
Purpose This paper is devoted to the theoretical and numerical study of a new topological sensitivity concerning the insertion of a small bolt connecting two parts in a mechanical structure. First, an idealized model of bolt is proposed which relies on a non-local interaction between the two ends of the bolt (head and threads) and possibly featuring a pre-stressed state. Second, a formula for the topological sensitivity of such an idealized bolt is rigorously derived for a large class of objective functions. Third, numerical tests are performed in $2 \mathrm{~d}$ and $3 \mathrm{~d}$ to assess the efficiency of the bolt topological sensitivity in the case of no pre-stress. In particular, the placement of bolts (acting then as springs) is coupled to the further optimization of their location and to the shape and topology of the structure for volume minimization under compliance constraint.

Design/methodology/approach The methodology relies on the adjoint method and the variational formulation of the linearized elasticity equations in order to establish the topological sensitivity.

Findings The numerical results prove the influence of the number and locations of the bolts which strongly influence the final optimized design of the structure.

Originality/value This paper is the first one to study the topology optimization of bolted systems without a fixed prescribed number of bolts.
\end{abstract}

Keywords Topological derivative, Bolt connection, Topology optimization 


\section{Contents}

1 Introduction $\quad 3$

2 Modeling $\quad 4$

2.1 Idealized bolt model . . . . . . . . . . . . . . . . . . . . . . . . . 4

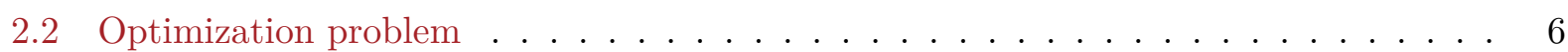

$\begin{array}{lll}3 & \text { Topological derivative and main results } & \mathbf{7}\end{array}$

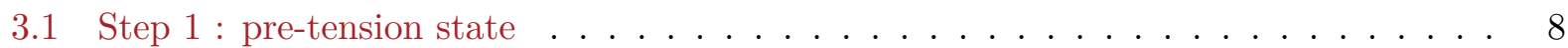

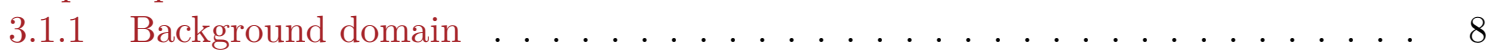

3.1 .2 Perturbed domain . . . . . . . . . . . . . . . . . . . 8

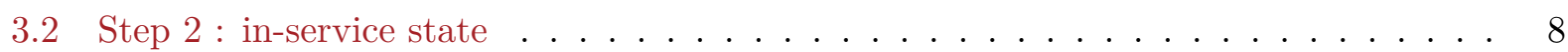

3.2 .1 Background domain . . . . . . . . . . . . . . . . . 8

3.2 .2 Perturbed domain . . . . . . . . . . . . . . . . . . . . 9

3.3 Main result . . . . . . . . . . . . . . . . . . . . . . . . . 9

4 Establishment of the topological derivative $\quad \mathbf{1 0}$

4.1 Approximation of perturbed displacement fields . . . . . . . . . . . . . . . . . . 10

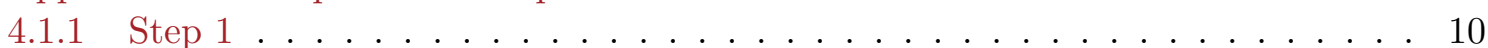

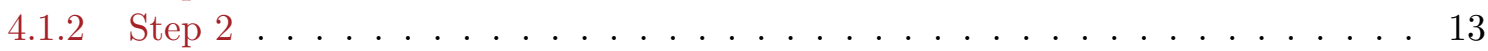

4.2 Approximation of the perturbed adjoint state . . . . . . . . . . . 16

4.3 Proof of Theorem $1 \ldots \ldots \ldots \ldots \ldots \ldots$

5 Optimization methods $\quad \mathbf{2 2}$

5.1 A coupled optimization problem . . . . . . . . . . . . . . . . 22

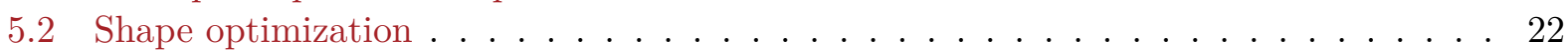

5.2 .1 Level-set method . . . . . . . . . . . . . . . . . . . . . . 22

5.2 .2 Hadamard's boundary variation method . . . . . . . . . . . . . . 22

5.3 Location optimization $\ldots \ldots \ldots \ldots \ldots \ldots \ldots$

6 Numerical illustrations $\quad \mathbf{2 4}$

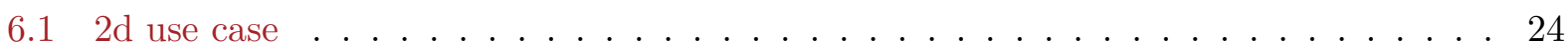

6.1.1 Description of the problem . . . . . . . . . . . . . . . 24

6.1.2 Test of the topological derivative . . . . . . . . . . . . . 25

6.1.3 Coupled optimization of both structure and springs location . . . . . . . . 26

6.1 .4 Optimization with one spring . . . . . . . . . . . . . 27

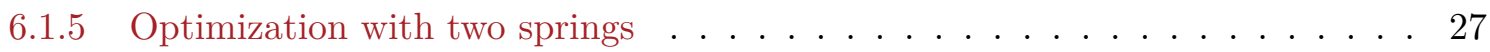

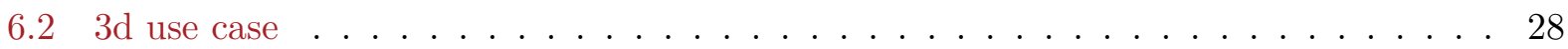

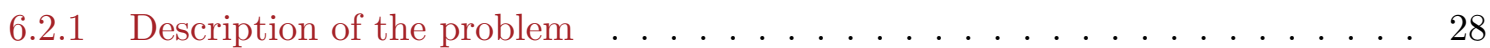

6.2.2 Search for the best location $x_{0}$ and orientation $e \ldots \ldots \ldots \ldots$

6.2.3 Fix the location $x_{0}$ and search for the best orientation $e \ldots \ldots \ldots$

6.2.4 Fix the orientation $e$ and search for the best location $x_{0} \ldots \ldots \ldots$ 


\section{Introduction}

Topological sensitivity analysis establishes a quantity called topological derivative which is a measure of the sensitivity of some objective function to an infinitesimally small perturbation of the topology of the system under study. It was first developed for a perturbation which is a small hole, with a Neumann boundary condition, in a structure governed by the linearized elasticity equations: its role is to determine if it is favorable or not to add a small hole in the structure during the optimization process, see $[11,12,26,28,29]$. Thereafter, the method has been applied to different type of boundary conditions on the edge of the hole, like Dirichlet boundary conditions for the Poisson's equation in [15] and for elasticity in [13]. The topological sensitivity approach was also extended to various other types of perturbation, like inclusions instead of holes, and to a wide range of models, such as sliding contact model for elasticity in [14], Navier-Stokes equations in [5], Helmholtz equation in [25], crack detection in [6], image processing in [7], inverse problems in [4], etc... The reader is referred to the textbook [18] for more examples and references.

To be more specific, the topological derivative is obtained by an asymptotic analysis of an objective function $J(\Omega)$, depending on a domain $\Omega$ in $\mathbb{R}^{n}(n=2$ or 3$)$, with respect to the insertion of a small hole or a small inclusion $\omega_{\rho} \subset \Omega$ with suitable boundary conditions. Given a reference shape $\omega$ (typically the unit ball), the small perturbation $\omega_{\rho}$ is centered at a point $\boldsymbol{x}_{0} \in \Omega$ and has a fixed shape $\omega$ that is rescaled by a small adimensional factor $\rho>0$. Define $J_{\rho}(\Omega)=J\left(\Omega \backslash \omega_{\rho}\right)$ the objective function for the perturbed domain, with the convention that $J_{0}(\Omega)=J(\Omega)$. The function $J_{\rho}$ is said to admit a topological derivative $D J\left(\boldsymbol{x}_{0}\right)$ at the point $\boldsymbol{x}_{0}$ for a hole or an inclusion of shape $\omega$, if the following asymptotic expansion holds for small $\rho>0$

$$
J_{\rho}(\Omega)=J_{0}(\Omega)+s(\rho) D J\left(\boldsymbol{x}_{0}\right)+o(s(\rho)),
$$

where $s$ is a positive real function of $\rho$ satisfying $\lim _{\rho \rightarrow 0} s(\rho)=0$. If the topological derivative $D J\left(\boldsymbol{x}_{0}\right)$ is negative, it is then favorable to create a small hole or a small inclusion at the point $\boldsymbol{x}_{0}$.

The goal of the present paper is to extend this definition to the insertion of a small bolt connecting two regions of a mechanical structure. Our idealization towards a thin bolt is both mechanically and geometrically driven. It is made of two small balls $\omega_{\rho}$ of size $\rho$, distant of a fixed length $\ell$ and aligned in a direction $\boldsymbol{e}$, connected by a spring of rigidity $\kappa(\rho)=K \rho^{k}$ subjected to a pre-stressed state, for some $k>1$ (see Fig. 1). Note that the length $\ell$ of the bolt is fixed, independent of $\rho$. The location of one of the balls $\omega_{\rho}$ is denoted by $\boldsymbol{x}_{0}$. Our main result in Subsection 3.3 is to derive a topological derivative $D J\left(\boldsymbol{x}_{0}, \boldsymbol{e}\right)$ for the sensitivity of an objective function $J(\Omega)$ upon insertion of a small bolt. Our proof of the validity of the topological asymptotic expansion in this case is not a simple generalization of previous studies in this field because of the fixed length of the bolt and the non-local coupling between the two end points of the bolt, $\kappa(\rho)$. Another original feature of our analysis is that the topological derivative $D J\left(\boldsymbol{x}_{0}, \boldsymbol{e}\right)$ depend on two variables, the location $\boldsymbol{x}_{0}$ and the orientation $e$ of the bolt.

Bolts are just one example of possible connections between different parts of a structure. Other possible types of connections are hinges, supports or rigid connections (a clamped or Dirichlet boundary conditions). Of course, the number and the locations of such mechanical connections have a dramatic impact on the performance of a structure. Therefore it makes sense to optimize, not only the location of connections (which can be done with a simple parametric optimization algorithm), but also their number: this task is performed by using the topological derivative which allows the addition of new connections where they are best used. The topological derivative concept has already been used to add simple connections like a clamped boundary or an embedded support condition. For instance, the topological derivative with respect to a small hole with Dirichlet boundary condition in linear elasticity is computed in [13]. In [8], a topological derivative approach is used to create new supports (or springs) in a discrete system of bars for optimizing its buckling load or one vibration eigenfrequency. The present paper is the first one to consider bolts in topology optimization: the topological derivative with respect to a small idealized bolt is used to find the 
best location and the optimal orientation of a new bolt. Some preliminary results were announced in our previous work [23], yet without proofs nor numerical illustrations for a coupled bolts and structure optimization. Part of this work was the topic of the PhD thesis of the first author [22] where other numerical examples can be found.

The contents of this paper are the followings. Section 2 defines the mechanical model for an idealized bolt: it is a two step process, where a first step induces a pre-stress before the second step of the normal operating mode of the tightened system. The mechanical model and the optimization problem are also set in Section 2. Section 3 introduces the perturbed domain for the topological sensitivity analysis with respect to a small bolt and gives our main theoretical results. Section 4 gives the proof of the formula for the topological derivative. Section 5 is devoted to the definition of a coupled optimization problem for the bolts and the structure. Once a new bolt has been introduced in a structure, thanks to the help of the topological derivative, its location can be optimized in a coupled manner with shape or topology optimization of the structure, using a parametric gradient-based algorithm and the level set method. Finally, Section 6 features numerical illustrations in $2 \mathrm{~d}$ and $3 \mathrm{~d}$ in the simplified case of no pre-stress in the bolt (in such a case the bolt is acting like a spring). For a $2 \mathrm{~d}$ academic example of volume minimization under a fixed compliance constraint, we explore various coupling strategies: optimizing the structure with a fixed bolt, initially placed by the topological derivative, or optimizing simultaneously the structure and the bolt location. The addition of a second bolt is also studied. In $3 \mathrm{~d}$ we content ourselves in testing the insertion of one or two bolts, without coupling with structure optimization. A more complete numerical assessment of our topological derivative in $3 \mathrm{~d}$ with engineering uses cases, featuring a pre-stressed bolt and a contact model, will appear in [24].

\section{Modeling}

\section{$2.1 \quad$ Idealized bolt model}

The purpose of a bolt is to connect two different parts of a structure. We propose here a simple setting to model the interface between these parts but more general and realistic cases can equally be considered (see Remark 1 below for a discussion). Let $\Omega$ be a smooth bounded and connected domain of $\mathbb{R}^{3}$, which is the union of two disjoint and connected parts $\Omega_{A}$ and $\Omega_{B}$, with an interface $\Gamma_{c}$ (see Fig. 2a). Its boundary is made of disjoint parts, $\partial \Omega=\Gamma \cup \Gamma_{N} \cup \Gamma_{D}$, where $\Gamma$ is the traction-free boundary and Neumann and Dirichlet boundary conditions are respectively imposed on $\Gamma_{N}$ and $\Gamma_{D}$, which is assumed to be non-empty. The space of admissible displacements corresponds to zero displacement on $\Gamma_{D}$

$$
\mathcal{W}=\left\{\boldsymbol{u} \in\left(H^{1}(\Omega)\right)^{3}, \boldsymbol{u}=\mathbf{0} \text { on } \Gamma_{D}\right\} .
$$

For a displacement field $\boldsymbol{u}$, the strain tensor is defined by $\varepsilon(\boldsymbol{u})=\frac{1}{2}\left(\nabla \boldsymbol{u}+\nabla^{T} \boldsymbol{u}\right)$. Each sub-domain $\Omega_{A}$ (resp. $\Omega_{B}$ ) is filled with a linear isotropic elastic material, with Lamé coefficients $\mu_{A}$ and $\lambda_{A}$ (resp. $\mu_{B}$ and $\lambda_{B}$ ). The stress tensor is then given by the Hooke's law

$$
\mathrm{A} \varepsilon(\boldsymbol{u})= \begin{cases}2 \mu_{A} \varepsilon(\boldsymbol{u})+\lambda_{A} \operatorname{tr}(\varepsilon(\boldsymbol{u})) \mathbf{I} & \text { in } \Omega_{A}, \\ 2 \mu_{B} \varepsilon(\boldsymbol{u})+\lambda_{B} \operatorname{tr}(\varepsilon(\boldsymbol{u})) \mathbf{I} & \text { in } \Omega_{B} .\end{cases}
$$

We assume a perfect interface $\Gamma_{c}$ between $\Omega_{A}$ and $\Omega_{B}$. Thus, there are usual transmission conditions between both parts that insure continuity of displacements and normal stress at the interface $\Gamma_{c}$. Assuming that there are only surface loads, the domain $\Omega$ is ruled by the linear elasticity equations

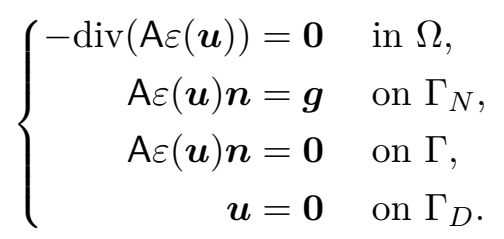


Assuming that surface loads $\boldsymbol{g}$ belong to $L^{2}\left(\Gamma_{N}\right)^{3}$, Lax-Milgram's theorem, coupled with Korn's inequality, gives the existence and the uniqueness of the solution $\boldsymbol{u} \in \mathcal{W}$. In the following, we assume that $\boldsymbol{u}$ is as smooth as we want, which is always possible by assuming that the loads $\boldsymbol{g}$ are smooth as well as the domain.

Remark 1. The assumption of a perfect interface between $\Omega_{A}$ and $\Omega_{B}$ is made here only for the sake of simplicity. Other settings are admissible for our analysis. Indeed, our main results do not depend directly on the type of conditions at the interface $\Gamma_{c}$. For example, one could consider that $\Gamma_{c}$ is a crack, where homogeneous Neumann boundary conditions hold true (ignoring possible inter-penetrations). In other words, $\Omega_{A}$ and $\Omega_{B}$ are two disconnected domains where the solutions of the linear elasticity equations can independently be computed (at least if both $\Omega_{A}$ and $\Omega_{B}$ have a Dirichlet boundary to insure well-posedness). One could further add a small gap between $\Omega_{A}$ and $\Omega_{B}$. Even more realistic is the case where a contact condition, instead of transmission condition, is imposed on $\Gamma_{c}$. This settings is studied in [22] Chapter 7, Section 7.3, where it is proved that a sliding contact condition between both parts does not change the final result as well as the analysis of the topological sensitivity. Of course, any hybrid combination of these conditions is possible, namely on different parts of $\Gamma_{c}$ different conditions hold true.

We propose an idealized model of bolt connections through an analytical formulation. Physical representativeness is kept at first order. This model aims to be computationally cheap and easy to use for optimization process.

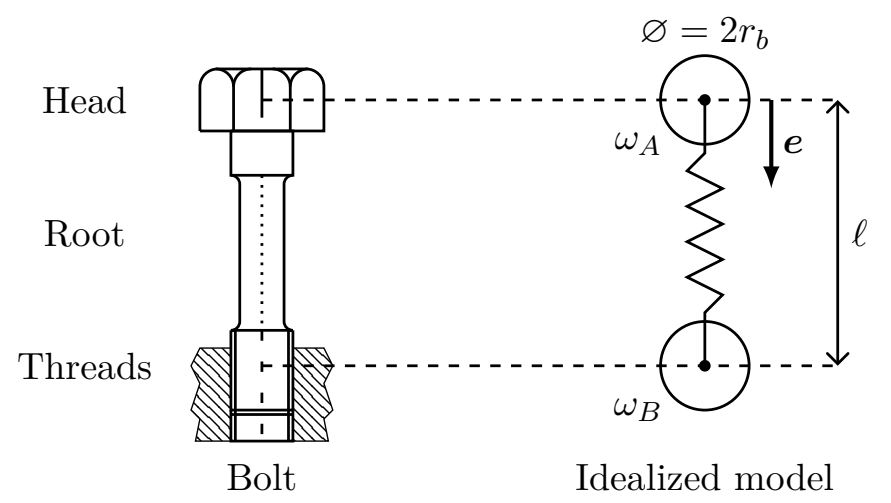

Figure 1: Standard bolt and its idealized model

Briefly stated, the idealized model corresponds to two spheres linked by a one degree of freedom linear-spring. The bolt is embodied by two spheres symbolizing its head and its threads, respectively denoted by $\omega_{A}$ and $\omega_{B}$ (see Fig. 1). These spheres allow efforts transmission and displacements continuity between the bolt extremities and the jointed parts. They stay at a distance $\ell$, in the direction of a unit vector $\boldsymbol{e}$, and have a radius $r_{b}$. We consider long bolts obeying to the EulerBernoulli condition for long beam which is $\ell \gg r_{b}$. The small bolt is obtained by rescaling the size of the spheres (but not the separation distance $\ell$ ) with an adimensional factor $\rho>0$ that goes to zero. In the following, the factor $\rho$ will abusively refer to the size of the bolt. The spheres are elastic, made of the same material as the parts. For the purpose of topological sensitivity analysis, the idealized model is associated to a rigidity $\kappa(\rho)$. It models the stiffness along the axis of the spring, that is to say its tension-compression behavior. The spheres remotely interact with each other through the linear spring law

$$
F=\kappa(\rho) L
$$

with $F$ the force and $L$ the lengthening of the bolt root.

Remark 2. An extension of this model with a 6 degrees of freedom linear-spring is described in [22] Chapter 2, Section 2.3. Such a model is irrelevant in the framework of topological sensitivity analysis which takes only into account the leading axial rigidity with regards to the size of the spheres. 
The bolt functioning with a pre-stress is described in two steps. In practice, a bolted joint is carried out by applying a tightening torque to the head of the bolt. It creates a pre-stressed state, denoted "Step 1". It consists in applying compressively an external pre-tension force of amplitude $\Phi(\rho)$ on the pair of elastic spheres (see Fig. 2a). This force corresponds to the compression of the tightened parts. For simplicity, this is performed in the absence of other external loads on the system. The second step, denoted "Step 2", amounts to add external loads $\boldsymbol{g}$ and the one degree of freedom spring of rigidity $\kappa(\rho)$ on this tightened system (see Fig. 2b). We denote by $T(\rho)$ the amplitude of the resulting force acting compressively between both spheres $\omega_{A}$ and $\omega_{B}$. That second step is described by a displacement field that depends on the displacement field of the first step too.

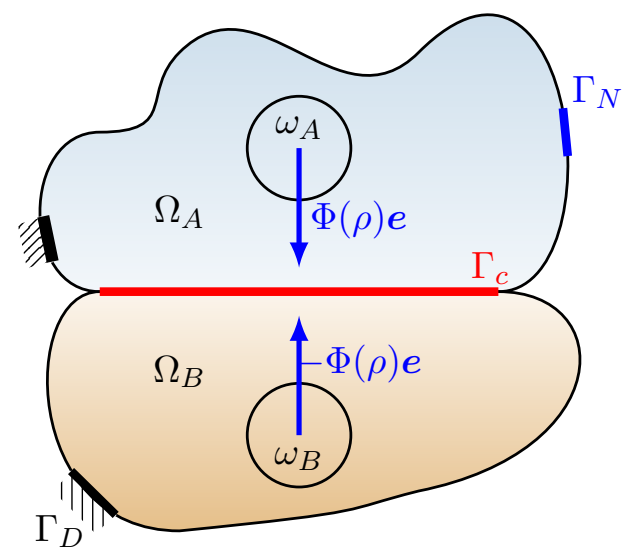

(a)

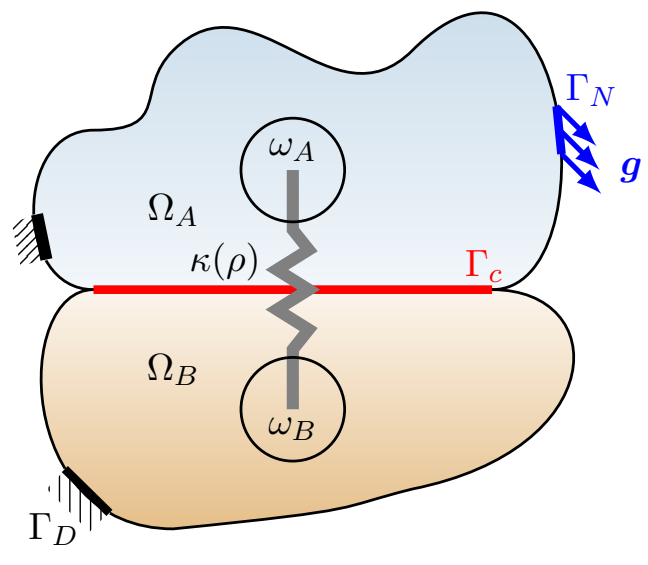

(b)

Figure 2: Step 1 : pre-stressed state (a) and Step 2 : in-service state where $\omega_{A}$ and $\omega_{B}$ are tightened (b)

\subsection{Optimization problem}

Let $\Omega_{1}$ and $\Omega_{2}$ be another partition of $\Omega$ (different from $\Omega_{A}$ and $\Omega_{B}$ ), that is $\bar{\Omega}=\bar{\Omega}_{1} \cup \bar{\Omega}_{2}$ and $\Omega_{1} \cap \Omega_{2}=\emptyset$. The goal of this partition is to avoid technicalities: bolts can be inserted in $\Omega_{2}$ by the topological derivative, away from the region $\Omega_{1}$ where the objective function is evaluated (see Fig. 3). This decoupling assumption simplifies the analysis, although it is not strictly necessary. The objective function is thus

$$
J_{0}(\Omega)=\int_{\Omega_{1}} F(\boldsymbol{u}) d V+\int_{\Gamma_{N}} G(\boldsymbol{u}) d S
$$

The functions $F$ and $G$ are twice differentiable and satisfy the growth conditions

$$
\exists \alpha>0,\left\{\begin{array}{l}
|F(\boldsymbol{u})| \leq \alpha\left(|\boldsymbol{u}|^{2}+1\right),\left|F^{\prime}(\boldsymbol{u})\right| \leq \alpha(|\boldsymbol{u}|+1),\left|F^{\prime \prime}(\boldsymbol{u})\right| \leq \alpha, \\
|G(\boldsymbol{u})| \leq \alpha\left(|\boldsymbol{u}|^{2}+1\right),\left|G^{\prime}(\boldsymbol{u})\right| \leq \alpha(|\boldsymbol{u}|+1),\left|G^{\prime \prime}(\boldsymbol{u})\right| \leq \alpha .
\end{array}\right.
$$

Knowing that the interface $\Gamma_{c}$ is fixed, we consider a shape optimization problem

$$
\min _{\Omega \in \mathcal{U}_{a d}} J_{0}(\Omega)
$$

where $\mathcal{U}_{a d}$ is the set of admissible shapes. In the absence of bolts, the computation of the gradient of the objective function (2.4) requires the introduction of the so-called background adjoint state $\boldsymbol{p}$, 
associated to (2.2), which solves

$$
\left\{\begin{aligned}
-\operatorname{div}(\mathrm{A} \varepsilon(\boldsymbol{p})) & =-\boldsymbol{F}^{\prime}(\boldsymbol{u}) \mathbb{1}_{\Omega_{1}} & & \text { in } \Omega, \\
\mathrm{A} \varepsilon(\boldsymbol{p}) \boldsymbol{n} & =-\boldsymbol{G}^{\prime}(\boldsymbol{u}) & & \text { on } \Gamma_{N}, \\
\mathrm{~A} \varepsilon(\boldsymbol{p}) \boldsymbol{n} & =-\mathbf{0} & & \text { on } \Gamma, \\
\boldsymbol{p} & =\mathbf{0} & & \text { on } \Gamma_{D},
\end{aligned}\right.
$$

where $\mathbb{1}_{\Omega_{1}}$ is the characteristic function of $\Omega_{1}$. Lax-Milgram's theorem, coupled with Korn's inequality, gives the existence and uniqueness of the solution $\boldsymbol{p} \in \mathcal{W}$. We assume that $\boldsymbol{p}$ is as smooth as required, similarly to the assumptions on $\boldsymbol{u}$.

\section{Topological derivative and main results}

We describe the insertion of a small bolt in the assembly with the topological derivative method. The background domain refers to the system before the insertion of the small idealized bolt. The perturbed domain refers to the domain with the new small bolt.

Remark 3. The analysis is still accurate and the expression of the topological derivative remains the same if the background domain already contains bolts (see [22] Chapter 7, Section 7.2, for the proof).

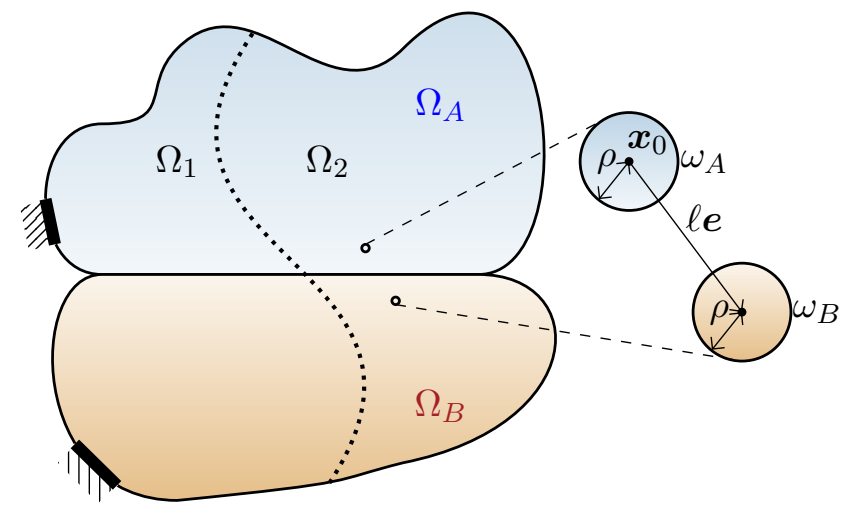

Figure 3: Perturbation of the domain $\Omega$ by a small bolt

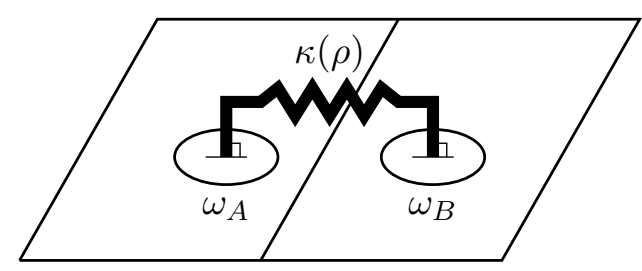

Figure 4: Sketch of the non-local rigidity $\kappa(\rho)$

We propose to perturb the background domain with two small elastic inclusions, $\omega_{A}$ and $\omega_{B}$ of size $\rho$, linked by a spring of rigidity $\kappa(\rho)$ subjected to a pre-tension of amplitude $\Phi(\rho)$. In the sequel, we choose the reference shape $\omega$ to be the unit ball of $\mathbb{R}^{3}$, which allows us to compute explicitly the coefficients of the topological derivative. Let $\omega_{A}$ be a small inclusion of shape $\omega$, rescaled by an adimensional factor $\rho>0$ and centred at the point $\boldsymbol{x}_{0} \in \Omega_{2}$. The second inclusion, denoted $\omega_{B}$, is the translation of $\omega_{A}$ at a distance $\ell>0$ and in the unit direction $\boldsymbol{e}$. More specifically, the inclusions read

$$
\omega_{A}=\left\{\boldsymbol{x} \in \mathbb{R}^{3}, \frac{\boldsymbol{x}-\boldsymbol{x}_{0}}{\rho} \in \omega\right\} \text { and } \omega_{B}=\left\{\boldsymbol{x} \in \mathbb{R}^{3}, \frac{\boldsymbol{x}-\boldsymbol{x}_{0}-\ell \boldsymbol{e}}{\rho} \in \omega\right\} .
$$

Let us assume the following scaling of the model

$$
\kappa(\rho)=K \rho^{k} \text { and } \Phi(\rho)=Q \rho^{k},
$$

with $K>0, Q>0$ and $k>1$. 
Remark 4. A complete analysis of all possible scalings is carried out in [22] Chapter 7, Section 7.2, where different powers are used for $\kappa(\rho)=K \rho^{k}, \Phi(\rho)=Q \rho^{q}, k>0$ and $q>0$.

The idealized bolt is defined with an averaged approach. Let us define the notation for the average on $\omega_{A}$ of the projection of the displacement field $\boldsymbol{u}$ along $\boldsymbol{e}$, the axis of the spring,

$$
f_{\omega_{A}} \boldsymbol{u} \cdot \boldsymbol{e} d V=\frac{1}{\left|\omega_{A}\right|} \int_{\omega_{A}} \boldsymbol{u} \cdot \boldsymbol{e} d V .
$$

The lengthening, $L$, of the spring is the difference between the average displacements in $\omega_{A}$ and $\omega_{B}$ along its axis. In $2 \mathrm{~d}$, this so-called spring can be depicted as an out-of-plane non-local rigidity linking the two spheres (cf. Fig. 4), acting as a remote interaction law. We define the perforated domain $\Omega_{\rho}$, i.e. the domain $\Omega$ without the head and the threads of the idealized bolt

$$
\Omega_{\rho}=\Omega \backslash\left(\omega_{A} \cup \omega_{B}\right) .
$$

\subsection{Step 1 : pre-tension state}

\subsubsection{Background domain}

For the sake of simplicity, it is assumed here that Step 1 takes place without any previous bolts and without external loads in background domain.

Thus, the background solution $\boldsymbol{u}_{1}$ is zero. However, as shown in [22] Chapter 7, Section 7.2, the entire analysis works also in the case of pre-existing bolts, yielding a non-zero background solution $\boldsymbol{u}_{1}$.

\subsubsection{Perturbed domain}

The background domain is perturbed by two small spheres loaded by a pre-tension force of amplitude $\Phi(\rho)$ (see Fig. 2a). The displacement field in the perturbed domain $\boldsymbol{u}_{\rho, 1}$ then solves

$$
\left\{\begin{aligned}
-\operatorname{div}\left(\mathrm{A} \varepsilon\left(\boldsymbol{u}_{\rho, 1}\right)\right) & =\mathbf{0} & & \text { in } \Omega_{\rho} \\
-\operatorname{div}\left(\mathrm{A} \varepsilon\left(\boldsymbol{u}_{\rho, 1}\right)\right) & =\frac{\Phi(\rho)}{\left|\omega_{A}\right|} \boldsymbol{e} & & \text { in } \omega_{A} \\
-\operatorname{div}\left(\mathrm{A} \varepsilon\left(\boldsymbol{u}_{\rho, 1}\right)\right) & =-\frac{\Phi(\rho)}{\left|\omega_{B}\right|} \boldsymbol{e} & & \text { in } \omega_{B} \\
\operatorname{A} \varepsilon\left(\boldsymbol{u}_{\rho, 1}\right) \boldsymbol{n} & =\mathbf{0} & & \text { on } \Gamma_{N} \\
\boldsymbol{u}_{\rho, 1} & =\mathbf{0} & & \text { on } \Gamma_{D}
\end{aligned}\right.
$$

Again, Lax-Milgram's theorem, with Korn's inequality, gives the existence and the uniqueness of the solution $\boldsymbol{u}_{\rho, 1} \in \mathcal{W}$. In the following, we assume that $\boldsymbol{u}_{\rho, 1}$ is smooth enough, at least away from $\omega_{A}$ and $\omega_{B}$ (the loads are discontinuous through the spheres boundaries). We also recall that the transmission condition between $\Omega_{A}$ and $\Omega_{B}$ insures the continuity of displacements and normal stress.

\subsection{Step 2 : in-service state}

\subsubsection{Background domain}

External loads $\boldsymbol{g}$ are applied in the second step. Then, the background solution $\boldsymbol{u}_{2}$ solves (2.2). 


\subsubsection{Perturbed domain}

The background problem (2.2) is perturbed by two small elastic spheres linked by a one degree of freedom spring of rigidity $\kappa(\rho)$ subjected to an apparent force $T(\rho)$ that reads

$$
T(\rho)=-\kappa(\rho)\left(f_{\omega_{B}} \boldsymbol{u}_{\rho, 1} \cdot \boldsymbol{e} d V-f_{\omega_{A}} \boldsymbol{u}_{\rho, 1} \cdot \boldsymbol{e} d V\right)+\Phi(\rho) .
$$

The displacement field $\boldsymbol{u}_{\rho, 2}$ solves

$$
\left\{\begin{array}{rlrl}
-\operatorname{div}\left(\mathrm{A} \varepsilon\left(\boldsymbol{u}_{\rho, 2}\right)\right) & =\mathbf{0} & \text { in } \Omega_{\rho} \\
-\operatorname{div}\left(\mathrm{A} \varepsilon\left(\boldsymbol{u}_{\rho, 2}\right)\right)-\frac{\kappa(\rho)}{\left|\omega_{A}\right|}\left(f_{\omega_{B}} \boldsymbol{u}_{\rho, 2} \cdot \boldsymbol{e} d V-f_{\omega_{A}} \boldsymbol{u}_{\rho, 2} \cdot \boldsymbol{e} d V\right) \boldsymbol{e} & =\frac{T(\rho)}{\left|\omega_{A}\right|} \boldsymbol{e} & \text { in } \omega_{A} \\
-\operatorname{div}\left(\mathrm{A} \varepsilon\left(\boldsymbol{u}_{\rho, 2}\right)\right)+\frac{\kappa(\rho)}{\left|\omega_{B}\right|}\left(f_{\omega_{B}} \boldsymbol{u}_{\rho, 2} \cdot \boldsymbol{e} d V-f_{\omega_{A}} \boldsymbol{u}_{\rho, 2} \cdot \boldsymbol{e} d V\right) \boldsymbol{e} & =-\frac{T(\rho)}{\left|\omega_{B}\right|} \boldsymbol{e} & & \text { in } \omega_{B} \\
\mathrm{~A} \varepsilon\left(\boldsymbol{u}_{\rho, 2}\right) \boldsymbol{n} & =\boldsymbol{g} & \text { on } \Gamma_{N} \\
\boldsymbol{u}_{\rho, 2} & =\mathbf{0} & \text { on } \Gamma_{D}
\end{array} .\right.
$$

Again, it is assumed that $\boldsymbol{u}_{\rho, 2}$ is as smooth as we want, at least away from $\omega_{A}$ and $\omega_{B}$, which is the case when the loads and the domain are smooth. Denote $J_{\rho}$ the objective function (2.4) evaluated in the perturbed domain

$$
J_{\rho}(\Omega)=\int_{\Omega_{1}} F\left(\boldsymbol{u}_{\rho, 2}\right) d V+\int_{\Gamma_{N}} G\left(\boldsymbol{u}_{\rho, 2}\right) d S .
$$

\subsection{Main result}

Definition 1. The objective function $J_{\rho}$ is said to admit a topological derivative $D J\left(\boldsymbol{x}_{0}, \boldsymbol{e}\right)$ at a point $\boldsymbol{x}_{0}$ for a small bolt of direction $\boldsymbol{e}$ and for a pair of inclusions of shape $\omega$, if the following asymptotic expansion holds for small $\rho>0$

$$
J_{\rho}(\Omega)=J_{0}(\Omega)+s(\rho) D J\left(\boldsymbol{x}_{0}, \boldsymbol{e}\right)+o(s(\rho)),
$$

where $s(\rho)$ is a positive scalar function of $\rho$ which satisfies $\lim _{\rho \rightarrow 0} s(\rho)=0$.

If the quantity $D J\left(\boldsymbol{x}_{0}, \boldsymbol{e}\right)$ is negative, it is then favorable to create a small bolt at the point $\boldsymbol{x}_{0}$ in the direction $\boldsymbol{e}$. Of course, in practice there may be some constraints in the optimization of $J_{\rho}$, like an extra weight of the bolt compared to the background material. In such a case, the topological derivative should be computed for the Lagrangian corresponding to this constrained optimization problem.

Remark 5. In the present study, the displacement field $\boldsymbol{u}_{1}$ of the background Step 1 is zero. Nevertheless, we give a general result in the following theorem which applies even if $\boldsymbol{u}_{1}$ does not vanish.

Theorem 1. Take $\omega$ to be the unit ball of $\mathbb{R}^{3}$. Define

$$
\begin{aligned}
& \boldsymbol{U}_{1}=\boldsymbol{u}_{1}\left(\boldsymbol{x}_{0}+\ell \boldsymbol{e}\right)-\boldsymbol{u}_{1}\left(\boldsymbol{x}_{0}\right), \\
& \boldsymbol{U}_{2}=\boldsymbol{u}_{2}\left(\boldsymbol{x}_{0}+\ell \boldsymbol{e}\right)-\boldsymbol{u}_{2}\left(\boldsymbol{x}_{0}\right), \\
& \boldsymbol{P}_{2}=\boldsymbol{p}_{2}\left(\boldsymbol{x}_{0}+\ell \boldsymbol{e}\right)-\boldsymbol{p}_{2}\left(\boldsymbol{x}_{0}\right),
\end{aligned}
$$


where $\boldsymbol{u}_{1}, \boldsymbol{u}_{2}$ and $\boldsymbol{p}_{2}$ are respectively the displacement fields of the background Step 1, Step 2 and the adjoint state of the background adjoint problem of Step 2. The objective function $J_{\rho}$ admits a topological asymptotic expansion of the form (3.8), for $k>1$, that reads

$$
J_{\rho}(\Omega)=J_{0}(\Omega)+\rho^{k}\left(K\left(\boldsymbol{U}_{2}-\boldsymbol{U}_{1}\right) \cdot \boldsymbol{e}+Q\right) \boldsymbol{P}_{2} \cdot \boldsymbol{e}+ \begin{cases}\mathcal{O}\left(\rho^{k+2}\right) & \text { if } k>4 \\ \mathcal{O}\left(\rho^{3 k / 2}\right) & \text { if } 2 \leq k \leq 4 \\ \mathcal{O}\left(\rho^{2 k-1}\right) & \text { if } 1<k<2\end{cases}
$$

Remark 6. The asymptotic expansion (3.10) of the objective function $J_{\rho}$ is the same whatever the transmission or boundary conditions at the interface $\Gamma_{c}$ between both parts $\Omega_{A}$ and $\Omega_{B}$. It is proved in [22] Chapter 7, Section 7.3, for the case of a sliding contact model on $\Gamma_{c}$. Note however that the condition on the interface $\Gamma_{c}$ appears implicitly in the result through the displacement fields and the adjoint. Nevertheless it does not change the coefficients of the topological derivative.

Corollary 1. In the absence of pre-tension force, in other words if $Q=0$, there is no pre-stressed state and the small bolt is degenerated into a simple spring. Therefore, the objective function $J_{\rho}$ admits a topological asymptotic expansion of the form (3.8), that reads

$$
J_{\rho}(\Omega)=J_{0}(\Omega)+\rho^{k} K \boldsymbol{U} \cdot \boldsymbol{e P} \cdot \boldsymbol{e}+ \begin{cases}\mathcal{O}\left(\rho^{k+2}\right) & \text { if } k>4 \\ \mathcal{O}\left(\rho^{3 k / 2}\right) & \text { if } 2 \leq k \leq 4 \\ \mathcal{O}\left(\rho^{2 k-1}\right) & \text { if } 1<k<2\end{cases}
$$

where $\boldsymbol{U}=\boldsymbol{u}\left(\boldsymbol{x}_{0}+\ell \boldsymbol{e}\right)-\boldsymbol{u}\left(\boldsymbol{x}_{0}\right)$ and $\boldsymbol{P}=\boldsymbol{p}\left(\boldsymbol{x}_{0}+\ell \boldsymbol{e}\right)-\boldsymbol{p}\left(\boldsymbol{x}_{0}\right)$ with $\boldsymbol{u}$ and $\boldsymbol{p}$ respectively solution of the background problem (2.2) and the associated adjoint problem (2.6).

Remark 7. Theorem 1 and Corollary 1 can be generalized to the 2d case (see [22] Chapter 5, Section 5.2, for details). For example, formula (3.11) becomes in $2 d$

$$
J_{\rho}(\Omega)=J_{0}(\Omega)+\rho^{k} K \boldsymbol{U} \cdot \boldsymbol{e P} \cdot \boldsymbol{e}+\left\{\begin{array}{ll}
\mathcal{O}\left(\rho^{k+2}\right) & \text { if } k \geq 4 \\
\mathcal{O}\left(\rho^{3 k / 2}\right) & \text { if } 0<k<4
\end{array} .\right.
$$

\section{Establishment of the topological derivative}

This section is devoted to the proof of Theorem 1. The main ideas are as follows. First, formal expansions (4.1), (4.9), (4.23) of the perturbed fields are established with the help of far field functions that zoom on each inclusion and ignore the boundary conditions on $\partial \Omega$. These far field functions are explicitly computed thanks to the spherical shape of the inclusions. Second, rigorous estimates are established to prove the validity of these asymptotic expansions. Third, these ansatz are used to compute the topological derivative, at the end of the in-service state (Step 2), from a Taylor expansion of the objective function. As usual, it requires the introduction of an adjoint, for which the same type of asymptotic analysis is required. One originality of the following proof is that the topological derivative is computed for a two steps process, involving two coupled state equations. In other words, the perturbed displacement field $\boldsymbol{u}_{\rho, 2}$ of Step 2, depends, among other things, on the displacement field $\boldsymbol{u}_{\rho, 1}$ of Step 1.

\subsection{Approximation of perturbed displacement fields}

\subsubsection{Step 1}

Recall that the background displacement field $\boldsymbol{u}_{1}$ of Step 1 is zero here. However, it is kept in the sequel to prove the general expression of the topological derivative. The following asymptotic 
expansion is formally proposed for the perturbed field $\boldsymbol{u}_{\rho, 1}$, solution of (3.4),

$$
\boldsymbol{u}_{\rho, 1}(\boldsymbol{x}) \approx \boldsymbol{u}_{1}+\boldsymbol{v}_{a, 1}\left(\frac{\boldsymbol{x}-\boldsymbol{x}_{0}}{\rho}\right)+\boldsymbol{v}_{b, 1}\left(\frac{\boldsymbol{x}-\boldsymbol{x}_{0}-\ell \boldsymbol{e}}{\rho}\right),
$$

where the far field functions $\boldsymbol{v}_{a, 1}$ and $\boldsymbol{v}_{b, 1}$ tackle the influence of each separate inclusions $\omega_{A}$ and $\omega_{B}$. They are defined in $\mathbb{R}^{3}$ and decay to zero at infinity since perturbations are getting smaller far from the inclusions. In other words, these functions are zooming on the inclusions and ignore boundary conditions on $\partial \Omega$. In (4.1) they are rescaled by $\rho$, so that $\boldsymbol{v}_{a, 1}$ and $\boldsymbol{v}_{b, 1}$ are living around the same unit size inclusion $\omega$, and respectively solve

$$
\left\{\begin{array} { l l } 
{ - \operatorname { d i v } ( \mathrm { A } \varepsilon ( \boldsymbol { v } _ { a , 1 } ) ( \boldsymbol { y } _ { a } ) ) = \mathbf { 0 } } & { \text { in } \mathbb { R } ^ { 3 } \backslash \omega } \\
{ - \operatorname { d i v } ( \mathrm { A } \varepsilon ( \boldsymbol { v } _ { a , 1 } ) ( \boldsymbol { y } _ { a } ) ) = \xi _ { u _ { 1 } } ( \rho ) \boldsymbol { e } } & { \text { in } \omega } \\
{ \operatorname { l i m } _ { \boldsymbol { y } _ { a } \rightarrow \mathbf { 0 } } \boldsymbol { v } _ { a , 1 } ( \boldsymbol { y } _ { a } ) = \mathbf { 0 } }
\end{array} \quad \text { and } \left\{\begin{array}{ll}
-\operatorname{div}\left(\mathrm{A} \varepsilon\left(\boldsymbol{v}_{b, 1}\right)\left(\boldsymbol{y}_{b}\right)\right)=\mathbf{0} & \text { in } \mathbb{R}^{3} \backslash \omega \\
-\operatorname{div}\left(\mathrm{A} \varepsilon\left(\boldsymbol{v}_{b, 1}\right)\left(\boldsymbol{y}_{b}\right)\right)=-\xi_{u_{1}}(\rho) \boldsymbol{e} & \text { in } \omega \\
\lim _{\boldsymbol{y}_{b} \rightarrow \mathbf{0}} \boldsymbol{v}_{b, 1}\left(\boldsymbol{y}_{b}\right)=\mathbf{0}
\end{array}\right.\right.
$$

where $\boldsymbol{y}_{a}=\frac{\boldsymbol{x}-\boldsymbol{x}_{0}}{\rho}, \boldsymbol{y}_{b}=\frac{\boldsymbol{x}-\boldsymbol{x}_{0}-\ell \boldsymbol{e}}{\rho}$ and

$$
\xi_{u_{1}}(\rho)=\frac{\Phi(\rho)}{\rho|\omega|} .
$$

Lemma 1. Assume that $\omega$ is a ball of radius $r_{1}=1$. Denoting $r=\|\boldsymbol{y}\|$, the functions $\boldsymbol{v}_{a, 1}$ and $\boldsymbol{v}_{b, 1}$ are explicitly given by

$$
\begin{gathered}
\boldsymbol{v}_{a, 1}(\boldsymbol{y})= \begin{cases}\frac{r_{1}^{3}\left(5 r^{2}-3 r_{1}^{2}\right)\left(\mu_{A}+\lambda_{A}\right)}{30 r^{5} \mu_{A}\left(2 \mu_{A}+\lambda_{A}\right)} \xi_{u_{1}}(\rho) \boldsymbol{e} \cdot \boldsymbol{y} \boldsymbol{y}+\frac{r_{1}^{3}\left(\left(15 \mu_{A}+5 \lambda_{A}\right) r^{2}+\left(\mu_{A}+\lambda_{A}\right) r_{1}^{2}\right)}{30 r^{3} \mu_{A}\left(2 \mu_{A}+\lambda_{A}\right)} \xi_{u_{1}}(\rho) \boldsymbol{e} & \text { in } \mathbb{R}^{3} \backslash \omega \\
\frac{\mu_{A}+\lambda_{A}}{15 \mu_{A}\left(2 \mu_{A}+\lambda_{A}\right)} \xi_{u_{1}}(\rho) \boldsymbol{e} \cdot \boldsymbol{y} \boldsymbol{y}-\frac{\left(9 \mu_{A}+4 \lambda_{A}\right) r^{2}-\left(25 \mu_{A}+10 \lambda_{A}\right) r_{1}^{2}}{30 \mu_{A}\left(2 \mu_{A}+\lambda_{A}\right)} \xi_{u_{1}}(\rho) \boldsymbol{e} & \text { in } \omega\end{cases} \\
\boldsymbol{v}_{b, 1}(\boldsymbol{y})=\left\{\begin{array}{ll}
-\frac{r_{1}^{3}\left(5 r^{2}-3 r_{1}^{2}\right)\left(\mu_{B}+\lambda_{B}\right)}{30 r^{5} \mu_{B}\left(2 \mu_{B}+\lambda_{B}\right)} \xi_{u_{1}}(\rho) \boldsymbol{e} \cdot \boldsymbol{y} \boldsymbol{y}-\frac{r_{1}^{3}\left(\left(15 \mu_{B}+5 \lambda_{B}\right) r^{2}+\left(\mu_{B}+\lambda_{B}\right) r_{1}^{2}\right)}{30 r^{3} \mu_{B}\left(2 \mu_{B}+\lambda_{B}\right)} \xi_{u_{1}}(\rho) \boldsymbol{e} & \text { in } \mathbb{R}^{3} \backslash \omega \\
-\frac{\mu_{B}+\lambda_{B}}{15 \mu_{B}\left(2 \mu_{B}+\lambda_{B}\right)} \xi_{u_{1}}(\rho) \boldsymbol{e} \cdot \boldsymbol{y} \boldsymbol{y}+\frac{\left(9 \mu_{B}+4 \lambda_{B}\right) r^{2}-\left(25 \mu_{B}+10 \lambda_{B}\right) r_{1}^{2}}{30 \mu_{B}\left(2 \mu_{B}+\lambda_{B}\right)} \xi_{u_{1}}(\rho) \boldsymbol{e} & \text { in } \omega
\end{array} .\right.
\end{gathered}
$$

Remark 8. In 2d, the solutions of the far fields problems (4.2) are obtained with the same methodology but they behave as $\mathcal{O}(\ln r)$ at infinity while they behave as $\mathcal{O}(1 / r)$ at infinity in $3 d$ (see [22] Chapter 5, Section 5.2.3).

Proof. The explicit form of the solutions of (4.2) goes back to [31]. A full proof can be found in [22] Chapter 5, Section 5.2. In the course of the computation, the following useful formula (for a later use) is obtained

$$
f_{\omega} \boldsymbol{v}_{a, 1} \cdot \boldsymbol{e} d V=\frac{\xi_{u_{1}}(\rho)}{\tau_{A}} \quad \text { with } \quad \tau_{A}=\frac{15}{2} \frac{\mu_{A}\left(2 \mu_{A}+\lambda_{A}\right)}{\left(5 \mu_{A}+2 \lambda_{A}\right) r_{1}^{2}},
$$

with the same formula for the average of $\boldsymbol{v}_{b, 1}$, replacing $A$ by $B$ and with a negative sign.

We now rigorously justify the ansatz (4.1). Let us introduce some simplifying notations. Denote by $\boldsymbol{v}_{\rho, 1}=\boldsymbol{u}_{\rho, 1}-\boldsymbol{u}_{1}$ the difference between the perturbed and the background displacement fields of Step 1. Since $\boldsymbol{u}_{1}$ is zero here, $\boldsymbol{v}_{\rho, 1}$ solves (3.4). Introduce the sum

$$
\boldsymbol{v}_{1}^{\rho}(\boldsymbol{x})=\boldsymbol{v}_{a, 1}\left(\frac{\boldsymbol{x}-\boldsymbol{x}_{0}}{\rho}\right)+\boldsymbol{v}_{b, 1}\left(\frac{\boldsymbol{x}-\boldsymbol{x}_{0}-\ell \boldsymbol{e}}{\rho}\right),
$$

where $\boldsymbol{v}_{a, 1}$ and $\boldsymbol{v}_{b, 1}$ are given by Lemma 1. The proposition below is a quantitative version of the asymptotic expansion (4.1). 
Proposition 1. Let $\theta \in \mathcal{C}_{c}^{\infty}(\Omega)$ be a cut-off function with compact support $\mathcal{U}_{+} \subset \Omega$ such that $\theta \equiv 1$ in a neighborhood $\mathcal{U}_{-} \subset \mathcal{U}_{+}$of $\boldsymbol{x}_{0}$ and $\boldsymbol{x}_{0}+\ell \boldsymbol{e}$. There exists a constant $C>0$ independent of $\rho$ such that

$$
\boldsymbol{v}_{\rho, 1}=\theta \boldsymbol{v}_{1}^{\rho}+\boldsymbol{\delta}_{1}^{\rho},
$$

with a small remainder term $\boldsymbol{\delta}_{1}^{\rho}$ satisfying

$$
\left\|\boldsymbol{v}_{1}^{\rho}\right\|_{L^{2}(\Omega)} \leq C \rho^{k}, \quad\left\|\varepsilon\left(\boldsymbol{v}_{1}^{\rho}\right)\right\|_{L^{2}(\Omega)} \leq C \rho^{k-1 / 2}, \quad\left\|\boldsymbol{\delta}_{1}^{\rho}\right\|_{H^{1}(\Omega)} \leq C \rho^{k} .
$$

Remark 9. The role of the cut-off function $\theta$ is to make sure that the product $\theta \boldsymbol{v}_{1}^{\rho}$ satisfies homogeneous boundary conditions on the boundary $\partial \Omega$, as does $\boldsymbol{v}_{\rho, 1}$. Since $\theta$ has a compact support, it implies that $\theta \equiv 0$ far from $\boldsymbol{x}_{0}$ and $\boldsymbol{x}_{0}+\ell \boldsymbol{e}$. Consequently, $\boldsymbol{\delta}_{1}^{\rho}$ also contains the far field influence of the inclusions. The function $\boldsymbol{\delta}_{1}^{\rho}$ is an error term in $H^{1}(\Omega)$ since its $H^{1}$-norm is always asymptotically smaller than the one of $\theta \boldsymbol{v}_{1}^{\rho}$. The $L^{2}$-norm of $\boldsymbol{\delta}_{1}^{\rho}$ is of the same order as its $H^{1}$-norm. However, the $L^{2}$-norm of $\boldsymbol{v}_{1}^{\rho}$ is always smaller by a factor $\sqrt{\rho}$ than the $L^{2}$-norm of its gradient $\varepsilon\left(\boldsymbol{v}_{1}^{\rho}\right)$.

Proof. The explicit expression of $\boldsymbol{v}_{a, 1}$ indicates at infinity that $\left|\boldsymbol{v}_{a, 1}\right|=\mathcal{O}\left(\left|\xi_{u_{1}}(\rho)\right| / r\right)$ and $\left|\varepsilon\left(\boldsymbol{v}_{a, 1}\right)\right|=$ $\mathcal{O}\left(\left|\xi_{u_{1}}(\rho)\right| / r^{2}\right)$. The same is true for $\boldsymbol{v}_{b, 1}$ and, by rescaling, it leads to

$$
\left\|\boldsymbol{v}_{1}^{\rho}\right\|_{L^{\infty}\left(\Omega \backslash \mathcal{U}_{-}\right)} \leq C \rho\left|\xi_{u_{1}}(\rho)\right| \quad \text { and } \quad\left\|\varepsilon\left(\boldsymbol{v}_{1}^{\rho}\right)\right\|_{L^{\infty}\left(\Omega \backslash \mathcal{U}_{-}\right)} \leq C \rho\left|\xi_{u_{1}}(\rho)\right| .
$$

Denote $\Omega_{\rho^{-1}}$ the translated and rescaled domain centered at the origin, equal to $\Omega_{\rho} / \rho$. Since $\boldsymbol{v}_{1}^{\rho}$ is the sum of two rescaled terms $\boldsymbol{v}_{a, 1}$ and $\boldsymbol{v}_{b, 1}$ which behave similarly, we have

$$
\begin{aligned}
\left\|\boldsymbol{v}_{1}^{\rho}\right\|_{L^{2}(\Omega)}^{2} & \leq C \rho^{3} \int_{\Omega_{\rho^{-1}}}\left|\boldsymbol{v}_{a, 1}\right|^{2} d V+C \rho^{3} \int_{\omega}\left|\boldsymbol{v}_{a, 1}\right|^{2} d V \leq C \rho^{3}\left|\xi_{u_{1}}(\rho)\right|^{2} \int_{r_{1}}^{r_{1} / \rho} d r+C \rho^{3}\left|\xi_{u_{1}}(\rho)\right|^{2} \int_{0}^{r_{1}} r^{6} d r \\
& \leq C \rho^{2}\left|\xi_{u_{1}}(\rho)\right|^{2}+C \rho^{3}\left|\xi_{u_{1}}(\rho)\right|^{2} \leq C \rho^{2}\left|\xi_{u_{1}}(\rho)\right|^{2} \leq C \Phi(\rho)^{2} \leq C \rho^{2 k} .
\end{aligned}
$$

Estimates of the $L^{2}$-norm of $\varepsilon\left(\boldsymbol{v}_{1}^{\rho}\right)$ are obtained by a similar argument

$$
\left\|\varepsilon\left(\boldsymbol{v}_{1}^{\rho}\right)\right\|_{L^{2}(\Omega)}^{2} \leq C \rho \int_{\Omega_{\rho^{-1}}}\left|\varepsilon\left(\boldsymbol{v}_{a, 1}\right)\right|^{2} d V+C \rho \int_{\omega}\left|\varepsilon\left(\boldsymbol{v}_{a, 1}\right)\right|^{2} d V \leq C \rho\left|\xi_{u_{1}}(\rho)\right|^{2} \leq C \frac{\Phi(\rho)^{2}}{\rho} \leq C \rho^{2 k-1} .
$$

To obtain an estimate of $\delta_{1}$, write its equation

$$
\left\{\begin{aligned}
-\operatorname{div}\left(\mathrm{A} \varepsilon\left(\boldsymbol{\delta}_{1}^{\rho}\right)\right) & =\operatorname{div} \mathrm{A}\left(\boldsymbol{v}_{1}^{\rho} \otimes \nabla \theta\right)^{s}+\mathrm{A} \varepsilon\left(\boldsymbol{v}_{1}^{\rho}\right) \nabla \theta & & \text { in } \Omega \\
\mathrm{A} \varepsilon\left(\boldsymbol{\delta}_{1}^{\rho}\right) \boldsymbol{n} & =\mathbf{0} & & \text { on } \Gamma_{N}, \\
\boldsymbol{\delta}_{1}^{\rho} & =\mathbf{0} & & \text { on } \Gamma_{D}
\end{aligned}\right.
$$

multiply it by $\boldsymbol{\delta}_{1}^{\rho}$ and integrate by parts

$$
\int_{\Omega} \mathrm{A} \varepsilon\left(\boldsymbol{\delta}_{1}^{\rho}\right): \varepsilon\left(\boldsymbol{\delta}_{1}^{\rho}\right) d V=-\int_{\Omega} \mathrm{A}\left(\boldsymbol{v}_{1}^{\rho} \otimes \nabla \theta\right)^{s}: \varepsilon\left(\boldsymbol{\delta}_{1}^{\rho}\right) d V+\int_{\Omega} \mathrm{A} \varepsilon\left(\boldsymbol{v}_{1}^{\rho}\right) \nabla \theta \cdot \boldsymbol{\delta}_{1}^{\rho} d V .
$$

The left hand side of (4.8) is bounded from below using the Poincaré-Korn inequality

$$
\int_{\Omega} \mathrm{A} \varepsilon\left(\boldsymbol{\delta}_{1}^{\rho}\right): \varepsilon\left(\boldsymbol{\delta}_{1}^{\rho}\right) d V \geq C\left\|\varepsilon\left(\boldsymbol{\delta}_{1}^{\rho}\right)\right\|_{L^{2}(\Omega)}^{2}
$$

for some constant $C$ independent of $\rho$. Using $L^{\infty}$-norms of $\boldsymbol{v}_{1}^{\rho}$ and $\varepsilon\left(\boldsymbol{v}_{1}^{\rho}\right)$ since $\nabla \theta=0$ in the influence area of the inclusions, the right hand side of (4.8) is bounded from above by

$$
\begin{aligned}
& C\left\|\boldsymbol{v}_{1}^{\rho}\right\|_{L^{\infty}\left(\Omega \backslash \mathcal{U}_{-}\right)}\left\|\varepsilon\left(\boldsymbol{\delta}_{1}^{\rho}\right)\right\|_{L^{2}(\Omega)}+C\left\|\varepsilon\left(\boldsymbol{v}_{1}^{\rho}\right)\right\|_{L^{\infty}\left(\Omega \backslash \mathcal{U}_{-}\right)}\left\|\boldsymbol{\delta}_{1}^{\rho}\right\|_{L^{2}(\Omega)} \\
& \leq C \rho\left|\xi_{u_{1}}(\rho)\right|\left\|\varepsilon\left(\boldsymbol{\delta}_{1}^{\rho}\right)\right\|_{L^{2}(\Omega)} \leq C \rho^{k}\left\|\varepsilon\left(\boldsymbol{\delta}_{1}^{\rho}\right)\right\|_{L^{2}(\Omega)},
\end{aligned}
$$

by using again the Poincaré-Korn inequality, which yields the desired result. 


\subsubsection{Step 2}

We perform a similar asymptotic analysis for the perturbed displacement field $\boldsymbol{u}_{\rho, 2}$, solution of (3.6), as we did for $\boldsymbol{u}_{\rho, 1}$. We propose the formal ansatz

$$
\boldsymbol{u}_{\rho, 2}(\boldsymbol{x}) \approx \boldsymbol{u}_{2}(\boldsymbol{x})+\boldsymbol{v}_{a, 2}\left(\frac{\boldsymbol{x}-\boldsymbol{x}_{0}}{\rho}\right)+\boldsymbol{v}_{b, 2}\left(\frac{\boldsymbol{x}-\boldsymbol{x}_{0}-\ell \boldsymbol{e}}{\rho}\right),
$$

where $\boldsymbol{v}_{a, 2}$ and $\boldsymbol{v}_{b, 2}$ are far field functions satisfying

$$
\left\{\begin{array} { l l } 
{ - \operatorname { d i v } ( \mathrm { A } \varepsilon ( \boldsymbol { v } _ { a , 2 } ) ( \boldsymbol { y } _ { a } ) ) = \mathbf { 0 } } & { \text { in } \mathbb { R } ^ { 3 } \backslash \omega } \\
{ - \operatorname { d i v } ( \mathrm { A } \varepsilon ( \boldsymbol { v } _ { a , 2 } ) ( \boldsymbol { y } _ { a } ) ) = \xi _ { u _ { 2 } } ( \rho ) \boldsymbol { e } } & { \text { in } \omega } \\
{ \operatorname { l i m } _ { \boldsymbol { y } _ { a } \rightarrow \mathbf { 0 } } \boldsymbol { v } _ { a , 2 } ( \boldsymbol { y } _ { a } ) = \mathbf { 0 } } & { }
\end{array} \text { and } \left\{\begin{array}{ll}
-\operatorname{div}\left(\mathrm{A} \varepsilon\left(\boldsymbol{v}_{b, 2}\right)\left(\boldsymbol{y}_{b}\right)\right)=\mathbf{0} & \text { in } \mathbb{R}^{3} \backslash \omega \\
-\operatorname{div}\left(\mathrm{A} \varepsilon\left(\boldsymbol{v}_{b, 2}\right)\left(\boldsymbol{y}_{b}\right)\right)=-\xi_{u_{2}}(\rho) \boldsymbol{e} & \text { in } \omega \\
\lim _{\boldsymbol{y}_{b} \rightarrow \mathbf{0}} \boldsymbol{v}_{b, 2}\left(\boldsymbol{y}_{b}\right)=\mathbf{0}
\end{array}\right.\right.
$$

with

$$
\begin{aligned}
\xi_{u_{2}}(\rho)= & \frac{\kappa(\rho)}{\rho|\omega|}\left(f_{\omega}\left(\boldsymbol{v}_{b, 2}-\boldsymbol{v}_{a, 2}\right) \cdot \boldsymbol{e} d V+\left(\boldsymbol{u}_{2}\left(\boldsymbol{x}_{0}+\ell \boldsymbol{e}\right)-\boldsymbol{u}_{2}\left(\boldsymbol{x}_{0}\right)\right) \cdot \boldsymbol{e}\right) \\
& -\frac{\kappa(\rho)}{\rho|\omega|}\left(f_{\omega}\left(\boldsymbol{v}_{b, 1}-\boldsymbol{v}_{a, 1}\right) \cdot \boldsymbol{e} d V+\left(\boldsymbol{u}_{1}\left(\boldsymbol{x}_{0}+\ell \boldsymbol{e}\right)-\boldsymbol{u}_{1}\left(\boldsymbol{x}_{0}\right)\right) \cdot \boldsymbol{e}\right)+\frac{\Phi(\rho)}{\rho|\omega|} .
\end{aligned}
$$

Because of the specific form (4.11) of the forcing term, the functions $\boldsymbol{v}_{a, 2}$ and $\boldsymbol{v}_{b, 2}$ are coupled together (which was not the case for $\boldsymbol{v}_{a, 1}$ and $\boldsymbol{v}_{b, 1}$ ). Furthermore, the forcing term depends on the solution at the first step. Indeed, the two last terms of (4.11) come from a zero-order expansion of the force $T(\rho)$ given by (3.5). They are simply obtained by using the ansatz (4.1) for $\boldsymbol{u}_{\rho, 1}$ and the Taylor expansions of the background solution $\boldsymbol{u}_{1}$ in a neighborhood of $\boldsymbol{x}_{0}$ and $\boldsymbol{x}_{0}+\ell \boldsymbol{e}$.

Remark 10. The coefficient $\xi_{u_{2}}(\rho)$ defined by (4.11) is not fully explicit since it depends on $\boldsymbol{v}_{a, 2}$ and $\boldsymbol{v}_{b, 2}$. However, as we shall see in the following lemma, it admits an explicit formula in terms of $\boldsymbol{u}_{1}$ and $\boldsymbol{u}_{2}$. In truth the notation $\xi_{u_{2}}(\rho)$ is slightly misleading since it does not depend solely on $\boldsymbol{u}_{2}$.

Lemma 2. Assume that $\omega$ is a ball of radius $r_{1}=1$. Denoting $r=\|\boldsymbol{y}\|$, the functions $\boldsymbol{v}_{a, 2}$ and $\boldsymbol{v}_{b, 2}$ are explicitly given by

$$
\begin{aligned}
& \boldsymbol{v}_{a, 2}(\boldsymbol{y})=\left\{\begin{array}{ll}
\frac{r_{1}^{3}\left(5 r^{2}-3 r_{1}^{2}\right)\left(\mu_{A}+\lambda_{A}\right)}{30 r^{5} \mu_{A}\left(2 \mu_{A}+\lambda_{A}\right)} \xi_{u_{2}}(\rho) \boldsymbol{e} \cdot \boldsymbol{y} \boldsymbol{y}+\frac{r_{1}^{3}\left(\left(15 \mu_{A}+5 \lambda_{A}\right) r^{2}+\left(\mu_{A}+\lambda_{A}\right) r_{1}^{2}\right)}{30 r^{3} \mu_{A}\left(2 \mu_{A}+\lambda_{A}\right)} \xi_{u_{2}}(\rho) \boldsymbol{e} & \text { in } \mathbb{R}^{3} \backslash \omega \\
\frac{\mu_{A}+\lambda_{A}}{15 \mu_{A}\left(2 \mu_{A}+\lambda_{A}\right)} \xi_{u_{2}}(\rho) \boldsymbol{e} \cdot \boldsymbol{y} \boldsymbol{y}-\frac{\left(9 \mu_{A}+4 \lambda_{A}\right) r^{2}-\left(25 \mu_{A}+10 \lambda_{A}\right) r_{1}^{2}}{30 \mu_{A}\left(2 \mu_{A}+\lambda_{A}\right)} \xi_{u_{2}}(\rho) \boldsymbol{e} & \text { in } \omega
\end{array},\right. \\
& \boldsymbol{v}_{b, 2}(\boldsymbol{y})=\left\{\begin{array}{ll}
-\frac{r_{1}^{3}\left(5 r^{2}-3 r_{1}^{2}\right)\left(\mu_{B}+\lambda_{B}\right)}{30 r^{5} \mu_{B}\left(2 \mu_{B}+\lambda_{B}\right)} \xi_{u_{2}}(\rho) \boldsymbol{e} \cdot \boldsymbol{y} \boldsymbol{y}-\frac{r_{1}^{3}\left(\left(15 \mu_{B}+5 \lambda_{B}\right) r^{2}+\left(\mu_{B}+\lambda_{B}\right) r_{1}^{2}\right)}{30 r^{3} \mu_{B}\left(2 \mu_{B}+\lambda_{B}\right)} \xi_{u_{2}}(\rho) \boldsymbol{e} & \text { in } \mathbb{R}^{3} \backslash \omega \\
-\frac{\mu_{B}+\lambda_{B}}{15 \mu_{B}\left(2 \mu_{B}+\lambda_{B}\right)} \xi_{u_{2}}(\rho) \boldsymbol{e} \cdot \boldsymbol{y} \boldsymbol{y}+\frac{\left(9 \mu_{B}+4 \lambda_{B}\right) r^{2}-\left(25 \mu_{B}+10 \lambda_{B}\right) r_{1}^{2}}{30 \mu_{B}\left(2 \mu_{B}+\lambda_{B}\right)} \xi_{u_{2}}(\rho) \boldsymbol{e} & \text { in } \omega
\end{array},\right.
\end{aligned}
$$

and

$$
\xi_{u_{2}}(\rho)=\frac{\frac{\kappa(\rho)}{\rho|\omega|}}{1+\frac{\kappa(\rho)}{\rho|\omega|}\left(\frac{1}{\tau_{A}}+\frac{1}{\tau_{B}}\right)}\left(\left(\boldsymbol{u}_{2}\left(\boldsymbol{x}_{0}+\ell \boldsymbol{e}\right)-\boldsymbol{u}_{2}\left(\boldsymbol{x}_{0}\right)\right) \cdot \boldsymbol{e}-\left(\boldsymbol{u}_{1}\left(\boldsymbol{x}_{0}+\ell \boldsymbol{e}\right)-\boldsymbol{u}_{1}\left(\boldsymbol{x}_{0}\right)\right) \cdot \boldsymbol{e}\right)+\frac{\Phi(\rho)}{\rho|\omega|} .
$$

Proof. The proof is as standard as the one of Lemma 1 thanks to [31]. Similar useful equalities hold and we have

$$
f_{\omega} \boldsymbol{v}_{a, 2} \cdot \boldsymbol{e} d V=\frac{\xi_{u_{2}}(\rho)}{\tau_{A}} \text { and } f_{\omega} \boldsymbol{v}_{b, 2} \cdot \boldsymbol{e} d V=-\frac{\xi_{u_{2}}(\rho)}{\tau_{B}} .
$$

The explicit expression of $\xi_{u_{2}}(\rho)$ is a combination of (4.11), (4.15) and stems from Lemma 1. 
Remark 11. Our choice (3.2) of scaling laws for $\kappa(\rho)=K \rho^{k}$ and $\Phi(\rho)=Q \rho^{k}$, with $k>1$, is partly motivated by the fact that both terms in definition (4.14) of $\xi_{u_{2}}(\rho)$ have the same order of magnitude and that asymptotically

$$
\xi_{u_{2}}(\rho)=\frac{\rho^{k-1}}{|\omega|}\left(K\left(\left(\boldsymbol{u}_{2}\left(\boldsymbol{x}_{0}+\ell \boldsymbol{e}\right)-\boldsymbol{u}_{2}\left(\boldsymbol{x}_{0}\right)\right) \cdot \boldsymbol{e}-\left(\boldsymbol{u}_{1}\left(\boldsymbol{x}_{0}+\ell \boldsymbol{e}\right)-\boldsymbol{u}_{1}\left(\boldsymbol{x}_{0}\right)\right) \cdot \boldsymbol{e}\right)+Q\right)+\mathcal{O}\left(\rho^{2 k-2}\right) .
$$

We now rigorously justify the ansatz (4.9). Let us introduce some simplifying notations. Define the sum

$$
\boldsymbol{v}_{2}^{\rho}(\boldsymbol{x})=\boldsymbol{v}_{a, 2}\left(\frac{\boldsymbol{x}-\boldsymbol{x}_{0}}{\rho}\right)+\boldsymbol{v}_{b, 2}\left(\frac{\boldsymbol{x}-\boldsymbol{x}_{0}-\ell \boldsymbol{e}}{\rho}\right),
$$

where $\boldsymbol{v}_{a, 2}$ and $\boldsymbol{v}_{b, 2}$ are given by Lemma 2. Let $\boldsymbol{v}_{\rho, 2}=\boldsymbol{u}_{\rho, 2}-\boldsymbol{u}_{2}$ be the difference between the perturbed and the background displacement fields of Step 2. It solves

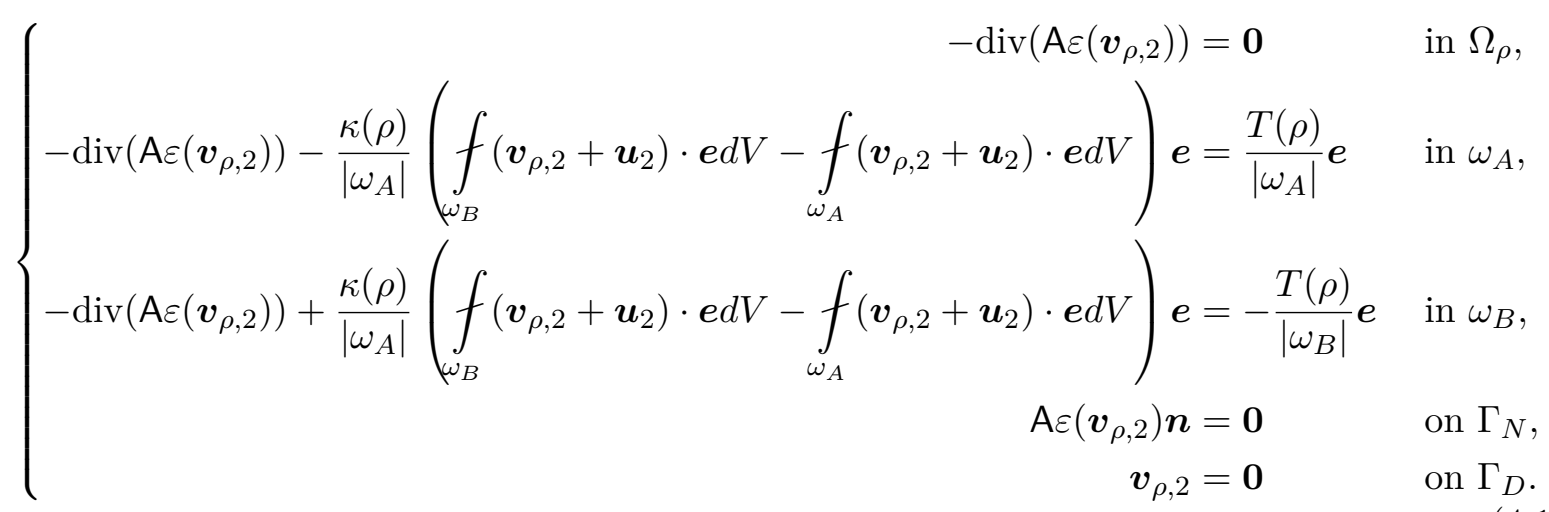

The proposition below gives a quantitative version of the asymptotic expansion (4.9) as $\rho$ goes to zero.

Proposition 2. Let $\theta \in \mathcal{C}_{c}^{\infty}(\Omega)$ be a cut-off function with compact support $\mathcal{U}_{+} \subset \Omega$ such that $\theta \equiv 1$ in a neighborhood $\mathcal{U}_{-} \subset \mathcal{U}_{+}$of $\boldsymbol{x}_{0}$ and $\boldsymbol{x}_{0}+\ell \boldsymbol{e}$. There exists a constant $C>0$ independent of $\rho$ such that

$$
\boldsymbol{v}_{\rho, 2}=\theta \boldsymbol{v}_{2}^{\rho}+\boldsymbol{\delta}_{2}^{\rho}
$$

with a small remainder term $\delta_{2}$ satisfying

$$
\left\|\boldsymbol{v}_{2}^{\rho}\right\|_{L^{2}(\Omega)} \leq C \rho^{k}, \quad\left\|\varepsilon\left(\boldsymbol{v}_{2}^{\rho}\right)\right\|_{L^{2}(\Omega)} \leq C \rho^{k-1 / 2}, \quad\left\|\boldsymbol{\delta}_{2}^{\rho}\right\|_{H^{1}(\Omega)} \leq C \rho^{k} .
$$

Proof. The explicit expression of $\boldsymbol{v}_{a, 2}$ gives its rate of decay at infinity, from which we deduce by rescaling $\left\|\boldsymbol{v}_{2}^{\rho}\right\|_{L^{\infty}\left(\Omega \backslash \mathcal{U}_{-}\right)} \leq C \rho\left|\xi_{u_{2}}(\rho)\right|$ and $\left\|\varepsilon\left(\boldsymbol{v}_{2}^{\rho}\right)\right\|_{L^{\infty}\left(\Omega \backslash \mathcal{U}_{-}\right)} \leq C \rho\left|\xi_{u_{2}}(\rho)\right|$. Using again the explicit form of $\boldsymbol{v}_{a, 2}$ and the scaling laws (3.2), as in the proof of Proposition 1, gives

$$
\begin{gathered}
\left\|\boldsymbol{v}_{2}^{\rho}\right\|_{L^{2}(\Omega)} \leq C \rho\left|\xi_{u_{2}}(\rho)\right| \leq C \frac{\kappa(\rho)}{1+C \frac{\kappa(\rho)}{\rho}}+C \Phi(\rho) \leq C \rho^{k}, \\
\left\|\varepsilon\left(\boldsymbol{v}_{2}^{\rho}\right)\right\|_{L^{2}(\Omega)} \leq C \sqrt{\rho}\left|\xi_{u_{2}}(\rho)\right| \leq C \frac{\frac{\kappa(\rho)}{\sqrt{\rho}}}{1+C \frac{\kappa(\rho)}{\rho}}+C \frac{\Phi(\rho)}{\sqrt{\rho}} \leq C \rho^{k-1 / 2} .
\end{gathered}
$$

Let us now write the equations satisfied by the error term $\delta_{2}$

$$
\left\{\begin{aligned}
-\operatorname{div}\left(\mathrm{A} \varepsilon\left(\boldsymbol{\delta}_{2}^{\rho}\right)\right) & =\operatorname{div} \mathrm{A}\left(\boldsymbol{v}_{2}^{\rho} \otimes \nabla \theta\right)^{s}+\mathrm{A} \varepsilon\left(\boldsymbol{v}_{2}^{\rho}\right) \nabla \theta & & \text { in } \Omega_{\rho}, \\
\mathrm{A} \varepsilon\left(\boldsymbol{\delta}_{2}^{\rho}\right) \boldsymbol{n} & =\mathbf{0} & & \text { on } \Gamma_{N}, \\
\boldsymbol{\delta}_{2}^{\rho} & =\mathbf{0} & & \text { on } \Gamma_{D},
\end{aligned}\right.
$$


complemented by the following equation in $\omega_{A}$

$$
\begin{aligned}
-\operatorname{div}\left(\mathrm{A} \varepsilon\left(\boldsymbol{\delta}_{2}^{\rho}\right)\right) & -\frac{\kappa(\rho)}{\left|\omega_{A}\right|}\left(f_{\omega_{B}}\left(\boldsymbol{\delta}_{2}^{\rho}-\boldsymbol{\delta}_{1}^{\rho}\right) \cdot \boldsymbol{e} d V-f_{\omega_{A}}\left(\boldsymbol{\delta}_{2}^{\rho}-\boldsymbol{\delta}_{1}^{\rho}\right) \cdot \boldsymbol{e} d V\right) \boldsymbol{e} \\
& =\frac{\kappa(\rho)}{\left|\omega_{A}\right|}\left(f_{\omega_{B}} \boldsymbol{u}_{2} \cdot \boldsymbol{e} d V-f_{\omega_{A}} \boldsymbol{u}_{2} \cdot \boldsymbol{e} d V-\left(\boldsymbol{u}_{2}\left(\boldsymbol{x}_{0}+\ell \boldsymbol{e}\right)-\boldsymbol{u}_{2}\left(\boldsymbol{x}_{0}\right)\right) \cdot \boldsymbol{e}\right) \boldsymbol{e} \\
& -\frac{\kappa(\rho)}{\left|\omega_{A}\right|}\left(f_{\omega_{B}} \boldsymbol{u}_{1} \cdot \boldsymbol{e} d V-f_{\omega_{A}} \boldsymbol{u}_{1} \cdot \boldsymbol{e} d V-\left(\boldsymbol{u}_{1}\left(\boldsymbol{x}_{0}+\ell \boldsymbol{e}\right)-\boldsymbol{u}_{1}\left(\boldsymbol{x}_{0}\right)\right) \cdot \boldsymbol{e}\right) \boldsymbol{e},
\end{aligned}
$$

and a similar one in $\omega_{B}$ obtained by interchanging the role of $\omega_{A}$ and $\omega_{B}$. To obtain an estimate of $\boldsymbol{\delta}_{2}^{\rho}$ we rely on the variational formulation of (4.18) and (4.19) with $\boldsymbol{\delta}_{2}^{\rho}$ as test function. Before doing so, let us recognize some simplifications in the right hand side of (4.19) by performing a Taylor expansion with integral remainder for $\boldsymbol{u}_{2}$ in a neighbourhood of $\boldsymbol{x}_{0}$

$$
\boldsymbol{u}_{2}(\boldsymbol{x})=\boldsymbol{u}_{2}\left(\boldsymbol{x}_{0}\right)+\nabla \boldsymbol{u}_{2}\left(\boldsymbol{x}_{0}\right)\left(\boldsymbol{x}-\boldsymbol{x}_{0}\right)+\int_{0}^{1}(1-t)\left(\boldsymbol{x}-\boldsymbol{x}_{0}\right)^{T} \mathcal{D}^{2}\left(\boldsymbol{u}_{2}\left(\boldsymbol{x}_{0}+t\left(\boldsymbol{x}-\boldsymbol{x}_{0}\right)\right)\right)\left(\boldsymbol{x}-\boldsymbol{x}_{0}\right) d t,
$$

where $\mathcal{D}^{2}\left(\boldsymbol{u}_{2}\right)$ is the Hessian tensor of the second order derivatives of $\boldsymbol{u}_{2}$. In particular, it is here that we need the assumption that $\boldsymbol{u}_{2}$ is smooth, at least $\boldsymbol{u}_{2} \in\left(\mathcal{C}^{2}(\Omega)\right)^{3}$. Recalling that $\omega$ is the unit ball of $\mathbb{R}^{3}$, it comes $f_{\omega_{A}} \nabla \boldsymbol{u}_{2}\left(\boldsymbol{x}_{0}\right)\left(\boldsymbol{x}-\boldsymbol{x}_{0}\right) d V=\mathbf{0}$ and thus

$$
f_{\omega_{A}} \boldsymbol{u}_{2} \cdot \boldsymbol{e} d V-\boldsymbol{u}_{2}\left(\boldsymbol{x}_{0}\right) \cdot \boldsymbol{e}=f_{\omega_{A}} \boldsymbol{R}_{u_{2}}^{A} d V
$$

where $\boldsymbol{R}_{u_{2}}^{A}$ denotes the integral remainder of the Taylor expansion (4.20). Similar functions, $\boldsymbol{R}_{u_{1}}^{A}$, $\boldsymbol{R}_{u_{1}}^{B}$ and $\boldsymbol{R}_{u_{2}}^{B}$, are set in a neighbourhood of $\boldsymbol{x}_{0}$ and $\boldsymbol{x}_{0}+\ell \boldsymbol{e}$. All these integral remainders are of the order of $\rho^{2}$ in the continuous norm of $\omega_{A}$ and $\omega_{B}$.

Eventually, the variational formulation of (4.18) and (4.19) with the test function $\boldsymbol{\delta}_{2}^{\rho}$, where the term $\frac{\kappa(\rho)}{4}\left(f_{\omega_{B}} \boldsymbol{\delta}_{1}^{\rho} \cdot \boldsymbol{e} d V-f_{\omega_{A}} \boldsymbol{\delta}_{1}^{\rho} \cdot \boldsymbol{e} d V\right)^{2}$ has been added on both sides, leads to

$$
\begin{aligned}
& \int_{\Omega} \mathrm{A} \varepsilon\left(\boldsymbol{\delta}_{2}^{\rho}\right): \varepsilon\left(\boldsymbol{\delta}_{2}^{\rho}\right) d V+\kappa(\rho)\left(f_{\omega_{B}} \boldsymbol{\delta}_{2}^{\rho} \cdot \boldsymbol{e} d V-f_{\omega_{A}} \boldsymbol{\delta}_{2}^{\rho} \cdot \boldsymbol{e} d V-\frac{1}{2}\left(f_{\omega_{B}} \boldsymbol{\delta}_{1}^{\rho} \cdot \boldsymbol{e} d V-f_{\omega_{A}} \boldsymbol{\delta}_{1}^{\rho} \cdot \boldsymbol{e} d V\right)\right)^{2} \\
= & -\int_{\Omega_{\rho}} \mathrm{A}\left(\boldsymbol{v}_{2}^{\rho} \otimes \nabla \theta\right)^{s}: \varepsilon\left(\boldsymbol{\delta}_{2}^{\rho}\right) d V+\int_{\Omega_{\rho}} \mathrm{A} \varepsilon\left(\boldsymbol{v}_{2}^{\rho}\right) \nabla \theta \cdot \boldsymbol{\delta}_{2}^{\rho} d V+\frac{\kappa(\rho)}{4}\left(f_{\omega_{B}} \boldsymbol{\delta}_{1}^{\rho} \cdot \boldsymbol{e} d V-f_{\omega_{A}} \boldsymbol{\delta}_{1}^{\rho} \cdot \boldsymbol{e} d V\right)^{2} \\
& -\kappa(\rho)\left(f_{\omega_{B}}\left(\boldsymbol{R}_{u_{2}}^{B}-\boldsymbol{R}_{u_{1}}^{B}\right) \cdot \boldsymbol{e} d V-f_{\omega_{A}}\left(\boldsymbol{R}_{u_{2}}^{A}-\boldsymbol{R}_{u_{1}}^{A}\right) \cdot \boldsymbol{e} d V\right)\left(f_{\omega_{B}} \boldsymbol{\delta}_{2}^{\rho} \cdot \boldsymbol{e} d V-f_{\omega_{A}} \boldsymbol{\delta}_{2}^{\rho} \cdot \boldsymbol{e} d V\right) .
\end{aligned}
$$

The left-hand side is as usual bounded from below with the Poincaré-Korn inequality

$$
\left|\int_{\Omega} \mathrm{A} \varepsilon\left(\boldsymbol{\delta}_{2}^{\rho}\right): \varepsilon\left(\boldsymbol{\delta}_{2}^{\rho}\right) d V\right| \geq C\left\|\varepsilon\left(\boldsymbol{\delta}_{2}^{\rho}\right)\right\|_{L^{2}(\Omega)}^{2} .
$$


The two first terms in the right-hand side are bounded from above, as previously, by

$$
C \rho\left|\xi_{u_{2}}(\rho)\right|\left\|\varepsilon\left(\boldsymbol{\delta}_{2}^{\rho}\right)\right\|_{L^{2}(\Omega)} \leq C \rho^{k}\left\|\varepsilon\left(\boldsymbol{\delta}_{2}^{\rho}\right)\right\|_{L^{2}(\Omega)} .
$$

The third term in the right-hand side is estimated by comparing the variational formulation of (3.4) with $\boldsymbol{\delta}_{1}^{\rho}$ as test function and the variational formulation of (4.7) with $\boldsymbol{v}_{\rho, 1}$ as test function. Since $\boldsymbol{v}_{\rho, 1}=\boldsymbol{u}_{\rho, 1}$, this comparison yields

$$
-\Phi(\rho)\left(f_{\omega_{B}} \boldsymbol{\delta}_{1}^{\rho} \cdot \boldsymbol{e} d V-f_{\omega_{A}} \boldsymbol{\delta}_{1}^{\rho} \cdot \boldsymbol{e} d V\right)=\int_{\Omega_{\rho}} \operatorname{divA}\left(\boldsymbol{v}_{1}^{\rho} \otimes \nabla \theta\right)^{s} \cdot \boldsymbol{v}_{\rho, 1} d V+\int_{\Omega_{\rho}} \mathrm{A} \varepsilon\left(\boldsymbol{v}_{1}^{\rho}\right) \nabla \theta \cdot \boldsymbol{v}_{\rho, 1} d V,
$$

the right hand side of which is bounded from above by

$$
C\left\|\nabla \boldsymbol{v}_{1}^{\rho}\right\|_{L^{\infty}\left(\Omega \backslash \mathcal{U}_{-}\right)}\left\|\boldsymbol{v}_{\rho, 1}\right\|_{L^{2}(\Omega)}+C\left\|\varepsilon\left(\boldsymbol{v}_{1}^{\rho}\right)\right\|_{L^{\infty}\left(\Omega \backslash \mathcal{U}_{-}\right)}\left\|\boldsymbol{v}_{\rho, 1}\right\|_{L^{2}(\Omega)} \leq C \rho^{2}\left|\xi_{u_{1}}\right|^{2} \leq C \Phi(\rho)^{2},
$$

since $\nabla \theta=0$ vanishes in $\mathcal{U}_{-}$. In particular, it implies that

$$
\left|f_{\omega_{B}} \boldsymbol{\delta}_{1}^{\rho} \cdot \boldsymbol{e} d V-f_{\omega_{A}} \boldsymbol{\delta}_{1}^{\rho} \cdot \boldsymbol{e} d V\right| \leq C \Phi(\rho) \leq C \rho^{k} .
$$

The fourth and last term on the right hand side is bounded from above by a combination of Cauchy-Schwarz inequality giving

$$
\left|f_{\omega_{B}} \boldsymbol{\delta}_{2}^{\rho} \cdot \boldsymbol{e} d V-f_{\omega_{A}} \boldsymbol{\delta}_{2}^{\rho} \cdot \boldsymbol{e} d V\right| \leq C \rho^{-3 / 2}\left\|\varepsilon\left(\boldsymbol{\delta}_{2}^{\rho}\right)\right\|_{L^{2}(\Omega)}
$$

and the properties of the integral remainder terms in $\omega_{A}$ and $\omega_{B}$ imply the estimate

$$
\left|f_{\omega_{B}}\left(\boldsymbol{R}_{u_{2}}^{B}-\boldsymbol{R}_{u_{1}}^{B}\right) \cdot \boldsymbol{e} d V-f_{\omega_{A}}\left(\boldsymbol{R}_{u_{2}}^{A}-\boldsymbol{R}_{u_{1}}^{A}\right) \cdot \boldsymbol{e d V}\right| \leq C \rho^{2} .
$$

Regrouping all terms finally leads to

$$
\left\|\varepsilon\left(\boldsymbol{\delta}_{2}^{\rho}\right)\right\|_{L^{2}(\Omega)}^{2} \leq C\left(\rho^{k}\left\|\varepsilon\left(\boldsymbol{\delta}_{2}^{\rho}\right)\right\|_{L^{2}(\Omega)}+\rho^{3 k}+\rho^{k+1 / 2}\left\|\varepsilon\left(\boldsymbol{\delta}_{2}^{\rho}\right)\right\|_{L^{2}(\Omega)}\right) \leq C \rho^{k}\left\|\varepsilon\left(\boldsymbol{\delta}_{2}^{\rho}\right)\right\|_{L^{2}(\Omega)}+C \rho^{3 k},
$$

from which it is easily deduced that $\left\|\varepsilon\left(\boldsymbol{\delta}_{2}^{\rho}\right)\right\|_{L^{2}(\Omega)} \leq C \rho^{k}$.

\subsection{Approximation of the perturbed adjoint state}

We now define an adjoint state $\boldsymbol{p}_{\rho, 2}$, associated to the perturbed displacement field $\boldsymbol{u}_{\rho, 2}(3.6)$ for the objective function $J_{\rho}$, defined by (3.7). The perturbed adjoint $\boldsymbol{p}_{\rho, 2}$ is the unique solution of

$$
\left\{\begin{aligned}
-\operatorname{div}\left(\mathrm{A} \varepsilon\left(\boldsymbol{p}_{\rho, 2}\right)\right) & =-\boldsymbol{F}^{\prime}\left(\boldsymbol{u}_{2}\right) \mathbb{1}_{\Omega_{2}} & & \text { in } \Omega_{\rho}, \\
-\operatorname{div}\left(\mathrm{A} \varepsilon\left(\boldsymbol{p}_{\rho, 2}\right)\right)-\frac{\kappa(\rho)}{\left|\omega_{A}\right|}\left(f_{\omega_{B}} \boldsymbol{p}_{\rho, 2} \cdot \boldsymbol{e} d V-f_{\omega_{A}} \boldsymbol{p}_{\rho, 2} \cdot \boldsymbol{e} d V\right) \boldsymbol{e} & =\mathbf{0} & & \text { in } \omega_{A}, \\
-\operatorname{div}\left(\mathrm{A} \varepsilon\left(\boldsymbol{p}_{\rho, 2}\right)\right)+\frac{\kappa(\rho)}{\left|\omega_{B}\right|}\left(f_{\omega_{B}} \boldsymbol{p}_{\rho, 2} \cdot \boldsymbol{e} d V-f_{\omega_{A}} \boldsymbol{p}_{\rho, 2} \cdot \boldsymbol{e} d V\right) \boldsymbol{e} & =\mathbf{0} & & \text { in } \omega_{B}, \\
\mathrm{~A} \varepsilon\left(\boldsymbol{p}_{\rho, 2}\right) \boldsymbol{n} & =-\boldsymbol{G}^{\prime}\left(\boldsymbol{u}_{2}\right) & & \text { on } \Gamma_{N} \\
\boldsymbol{p}_{\rho, 2} & =\mathbf{0} & & \text { on } \Gamma_{D} .
\end{aligned}\right.
$$

As for the perturbed displacement field $\boldsymbol{u}_{\rho, 2}$, we assume that the perturbed adjoint state $\boldsymbol{p}_{\rho, 2}$ is smooth, at least away from the inclusions $\omega_{A}$ and $\omega_{B}$. 
Remark 12. The adjoint problem is adapted to the sensitivity analysis. It has already been noticed in [3] that the right hand side of (4.22) depends on $\boldsymbol{u}_{2}$ and not on $\boldsymbol{u}_{\rho, 2}$ as expected in the perturbed domain. Moreover, the adjoint state is not directly affected by the pre-tension state $\boldsymbol{u}_{1}$.

We perform another asymptotic analysis for the perturbed adjoint, solution of (3.6), as we did for $\boldsymbol{u}_{\rho, 1}$ and $\boldsymbol{u}_{\rho, 2}$. We propose the formal ansatz

$$
\boldsymbol{p}_{\rho, 2}(\boldsymbol{x}) \approx \boldsymbol{p}_{2}(\boldsymbol{x})+\boldsymbol{q}_{a, 2}\left(\frac{\boldsymbol{x}-\boldsymbol{x}_{0}}{\rho}\right)+\boldsymbol{q}_{b, 2}\left(\frac{\boldsymbol{x}-\boldsymbol{x}_{0}-\ell \boldsymbol{e}}{\rho}\right),
$$

where $\boldsymbol{q}_{a, 2}$ and $\boldsymbol{q}_{b, 2}$ are far field functions satisfying

$$
\left\{\begin{array} { l l } 
{ - \operatorname { d i v } ( \mathrm { A } \varepsilon ( \boldsymbol { q } _ { a , 2 } ) ( \boldsymbol { y } _ { a } ) ) = \mathbf { 0 } } & { \text { in } \mathbb { R } ^ { 3 } \backslash \omega } \\
{ - \operatorname { d i v } ( \mathrm { A } \varepsilon ( \boldsymbol { q } _ { a , 2 } ) ( \boldsymbol { y } _ { a } ) ) = \xi _ { p _ { 2 } } ( \rho ) \boldsymbol { e } } & { \text { in } \omega } \\
{ \operatorname { l i m } _ { \boldsymbol { y } _ { a } \rightarrow \mathbf { 0 } } \boldsymbol { q } _ { a , 2 } ( \boldsymbol { y } _ { a } ) = \mathbf { 0 } } & { }
\end{array} \text { and } \left\{\begin{array}{ll}
-\operatorname{div}\left(\mathrm{A} \varepsilon\left(\boldsymbol{q}_{b, 2}\right)\left(\boldsymbol{y}_{b}\right)\right)=\mathbf{0} & \text { in } \mathbb{R}^{3} \backslash \omega \\
-\operatorname{div}\left(\mathrm{A} \varepsilon\left(\boldsymbol{q}_{b, 2}\right)\left(\boldsymbol{y}_{b}\right)\right)=-\xi_{p_{2}}(\rho) \boldsymbol{e} & \text { in } \omega \\
\lim _{\boldsymbol{y}_{b} \rightarrow \mathbf{0}} \boldsymbol{q}_{b, 2}\left(\boldsymbol{y}_{b}\right)=\mathbf{0}
\end{array}\right.\right.
$$

with

$$
\xi_{p_{2}}(\rho)=\frac{\frac{\kappa(\rho)}{\rho|\omega|}}{1+\frac{\kappa(\rho)}{\rho|\omega|}\left(\frac{1}{\tau_{A}}+\frac{1}{\tau_{B}}\right)} \boldsymbol{P}_{2} \cdot \boldsymbol{e} .
$$

To rigorously justify the ansatz (4.23) we introduce the notations $\boldsymbol{q}_{\rho, 2}=\boldsymbol{p}_{\rho, 2}(\boldsymbol{x})-\boldsymbol{p}_{2}(\boldsymbol{x})$ and $\boldsymbol{q}_{2}^{\rho}(\boldsymbol{x})=\boldsymbol{q}_{a, 2}\left(\frac{\boldsymbol{x}-\boldsymbol{x}_{0}}{\rho}\right)+\boldsymbol{q}_{b, 2}\left(\frac{\boldsymbol{x}-\boldsymbol{x}_{0}-\ell \boldsymbol{e}}{\rho}\right)$.

Proposition 3. Let $\theta \in \mathcal{C}_{c}^{\infty}(\Omega)$ be a cut-off function with compact support $\mathcal{U}_{+} \subset \Omega$ such that $\theta \equiv 1$ in a neighbourhood $\mathcal{U}_{-} \subset \mathcal{U}_{+}$of $\boldsymbol{x}_{0}$ and $\boldsymbol{x}_{0}+\ell \boldsymbol{e}$. We have

$$
\boldsymbol{q}_{\rho, 2}=\theta \boldsymbol{q}_{2}^{\rho}+\boldsymbol{\eta}_{2}^{\rho},
$$

where $\boldsymbol{\eta}_{2}^{\rho}$ is a small remainder term satisfying

$$
\left\|\boldsymbol{q}_{2}^{\rho}\right\|_{L^{2}(\Omega)} \leq C \rho^{k}, \quad\left\|\varepsilon\left(\boldsymbol{q}_{2}^{\rho}\right)\right\|_{L^{2}(\Omega)} \leq C \rho^{k-1 / 2}, \quad\left\|\boldsymbol{\eta}_{2}^{\rho}\right\|_{H^{1}(\Omega)} \leq C \rho^{k} .
$$

Proof. The error term $\boldsymbol{\eta}_{2}^{\rho}$ solves

$$
\left\{\begin{aligned}
-\operatorname{div}\left(\mathrm{A} \varepsilon\left(\boldsymbol{\eta}_{2}^{\rho}\right)\right) & =\operatorname{divA}\left(\boldsymbol{q}_{2}^{\rho} \otimes \nabla \theta\right)^{s}+\mathrm{A} \varepsilon\left(\boldsymbol{q}_{2}^{\rho}\right) \nabla \theta & & \text { in } \Omega_{\rho} \\
\mathrm{A} \varepsilon\left(\boldsymbol{\eta}_{2}^{\rho}\right) \boldsymbol{n} & =\mathbf{0} & & \text { on } \Gamma_{N} \\
\boldsymbol{\eta}_{2}^{\rho} & =\mathbf{0} & & \text { on } \Gamma_{D} .
\end{aligned}\right.
$$

complemented with the following equation in $\omega_{A}$

$$
\begin{aligned}
-\operatorname{div}\left(\mathrm{A} \varepsilon\left(\boldsymbol{\eta}_{2}^{\rho}\right)\right) & -\frac{\kappa(\rho)}{\left|\omega_{A}\right|}\left(f_{\omega_{B}} \boldsymbol{\eta}_{2}^{\rho} \cdot \boldsymbol{e} d V-f_{\omega_{A}} \boldsymbol{\eta}_{2}^{\rho} \cdot \boldsymbol{e} d V\right) \boldsymbol{e} \\
& =\frac{\kappa(\rho)}{\left|\omega_{A}\right|}\left(f_{\omega_{B}} \boldsymbol{p}_{2} \cdot \boldsymbol{e} d V-f_{\omega_{A}} \boldsymbol{p}_{2} \cdot \boldsymbol{e} d V-\left(\boldsymbol{p}_{2}\left(\boldsymbol{x}_{0}+\ell \boldsymbol{e}\right)-\boldsymbol{p}_{2}\left(\boldsymbol{x}_{0}\right)\right) \cdot \boldsymbol{e}\right) \boldsymbol{e}
\end{aligned}
$$

and a similar one in $\omega_{B}$ obtained by interchanging the role of $\omega_{A}$ and $\omega_{B}$. Following the ingredients of the proof of Proposition 2, substituting $\boldsymbol{u}_{2}$ with $\boldsymbol{p}_{2}$ and $\boldsymbol{v}_{\rho, 2}$ with $\boldsymbol{q}_{\rho, 2}$ and not considering the pre-stressed state i.e. taking $T(\rho)=0$, lead to the desired conclusion. 


\subsection{Proof of Theorem 1}

We perform a Taylor expansion with exact remainder of the objective function (3.7)

$J_{\rho}(\Omega)=J_{0}(\Omega)+\int_{\Omega_{1}} \boldsymbol{F}^{\prime}\left(\boldsymbol{u}_{2}\right) \cdot \boldsymbol{v}_{\rho, 2} d V+\int_{\Gamma_{N}} \boldsymbol{G}^{\prime}\left(\boldsymbol{u}_{2}\right) \cdot \boldsymbol{v}_{\rho, 2} d S+\frac{1}{2} \int_{\Omega_{1}} \boldsymbol{v}_{\rho, 2}^{T} F^{\prime \prime}\left(\overline{\boldsymbol{u}_{2}}\right) \boldsymbol{v}_{\rho, 2} d V+\frac{1}{2} \int_{\Gamma_{N}} \boldsymbol{v}_{\rho, 2}^{T} G^{\prime \prime}\left(\overline{\boldsymbol{u}_{2}}\right) \boldsymbol{v}_{\rho, 2} d S$.

In view of assumption (2.5) and the scaling laws (3.2), the exact remainder terms are bounded by

$$
\left|\int_{\Omega_{1}} \boldsymbol{v}_{\rho, 2}^{T} F^{\prime \prime}\left(\overline{\boldsymbol{u}_{2}}\right) \boldsymbol{v}_{\rho, 2} d V\right|+\left|\int_{\Gamma_{N}} \boldsymbol{v}_{\rho, 2}^{T} G^{\prime \prime}(\overline{\boldsymbol{u}}) \boldsymbol{v}_{\rho, 2} d S\right| \leq C \rho^{2}\left|\xi_{u_{2}}(\rho)\right|^{2} \leq C \rho^{2 k} .
$$

Multiplying the adjoint system (4.22) by $\boldsymbol{v}_{\rho, 2}$ and comparing to system (4.17) multiplied by the adjoint $\boldsymbol{p}_{\rho, 2}$, we deduce

$$
\begin{aligned}
& \int_{\Omega_{1}} \boldsymbol{F}^{\prime}\left(\boldsymbol{u}_{2}\right) \cdot \boldsymbol{v}_{\rho, 2} d V+\int_{\Gamma_{N}} \boldsymbol{G}^{\prime}\left(\boldsymbol{u}_{2}\right) \cdot \boldsymbol{v}_{\rho, 2} d S \\
= & \left(\kappa(\rho)\left(f_{\omega_{B}}\left(\boldsymbol{u}_{2}-\boldsymbol{u}_{\rho, 1}\right) \cdot \boldsymbol{e} d V-f_{\omega_{A}}\left(\boldsymbol{u}_{2}-\boldsymbol{u}_{\rho, 1}\right) \cdot \boldsymbol{e} d V\right)+\Phi(\rho)\right)\left(f_{\omega_{B}} \boldsymbol{p}_{\rho, 2} \cdot \boldsymbol{e} d V-f_{\omega_{A}} \boldsymbol{p}_{\rho, 2} \cdot \boldsymbol{e} d V\right) .
\end{aligned}
$$

It remains to replace the $\rho$-dependent functions by their ansatz, $\boldsymbol{u}_{\rho, 1}=\boldsymbol{u}_{1}+\theta \boldsymbol{v}_{1}^{\rho}+\boldsymbol{\delta}_{1}^{\rho}$ and $\boldsymbol{p}_{\rho, 2}=\boldsymbol{p}_{2}+\theta \boldsymbol{q}_{2}^{\rho}+\boldsymbol{\eta}_{2}^{\rho}$. The topological derivative and the remainder term are then evaluated by 
estimating the following 9 terms (one by line)

$$
\begin{aligned}
& \int_{\Omega_{1}} \boldsymbol{F}^{\prime}\left(\boldsymbol{u}_{2}\right) \cdot \boldsymbol{v}_{\rho, 2} d V+\int_{\Gamma_{N}} \boldsymbol{G}^{\prime}\left(\boldsymbol{u}_{2}\right) \cdot \boldsymbol{v}_{\rho, 2} d S \\
& =\left(\kappa(\rho)\left(f_{\omega_{B}}\left(\boldsymbol{u}_{2}-\boldsymbol{u}_{1}\right) \cdot \boldsymbol{e} d V-f_{\omega_{A}}\left(\boldsymbol{u}_{2}-\boldsymbol{u}_{1}\right) \cdot \boldsymbol{e} d V\right)+\Phi(\rho)\right)\left(f_{\omega_{B}} \boldsymbol{p}_{2} \cdot \boldsymbol{e} d V-f_{\omega_{A}} \boldsymbol{p}_{2} \cdot \boldsymbol{e} d V\right) \\
& +\left(\kappa(\rho)\left(f_{\omega_{B}}\left(\boldsymbol{u}_{2}-\boldsymbol{u}_{1}\right) \cdot \boldsymbol{e} d V-f_{\omega_{A}}\left(\boldsymbol{u}_{2}-\boldsymbol{u}_{1}\right) \cdot \boldsymbol{e} d V\right)+\Phi(\rho)\right)\left(f_{\omega_{B}} \boldsymbol{q}_{2}^{\rho} \cdot \boldsymbol{e} d V-f_{\omega_{A}} \boldsymbol{q}_{2}^{\rho} \cdot \boldsymbol{e} d V\right) \\
& +\left(\kappa(\rho)\left(f_{\omega_{B}}\left(\boldsymbol{u}_{2}-\boldsymbol{u}_{1}\right) \cdot \boldsymbol{e} d V-f_{\omega_{A}}\left(\boldsymbol{u}_{2}-\boldsymbol{u}_{1}\right) \cdot \boldsymbol{e} d V\right)+\Phi(\rho)\right)\left(f_{\omega_{B}} \boldsymbol{\eta}_{2}^{\rho} \cdot \boldsymbol{e} d V-f_{\omega_{A}} \boldsymbol{\eta}_{2}^{\rho} \cdot \boldsymbol{e} d V\right) \\
& -\kappa(\rho)\left(f_{\omega_{B}} \boldsymbol{v}_{1}^{\rho} \cdot \boldsymbol{e} d V-f_{\omega_{A}} \boldsymbol{v}_{1}^{\rho} \cdot \boldsymbol{e} d V\right)\left(f_{\omega_{B}} \boldsymbol{p}_{2} \cdot \boldsymbol{e} d V-f_{\omega_{A}} \boldsymbol{p}_{2} \cdot \boldsymbol{e} d V\right) \\
& -\kappa(\rho)\left(f_{\omega_{B}} \boldsymbol{v}_{1}^{\rho} \cdot \boldsymbol{e} d V-f_{\omega_{A}} \boldsymbol{v}_{1}^{\rho} \cdot \boldsymbol{e} d V\right)\left(f_{\omega_{B}} \boldsymbol{q}_{2}^{\rho} \cdot \boldsymbol{e} d V-f_{\omega_{A}} \boldsymbol{q}_{2}^{\rho} \cdot \boldsymbol{e} d V\right) \\
& -\kappa(\rho)\left(f_{\omega_{B}} \boldsymbol{v}_{1}^{\rho} \cdot \boldsymbol{e} d V-f_{\omega_{A}} \boldsymbol{v}_{1}^{\rho} \cdot \boldsymbol{e} d V\right)\left(f_{\omega_{B}} \boldsymbol{\eta}_{2}^{\rho} \cdot \boldsymbol{e} d V-f_{\omega_{A}} \boldsymbol{\eta}_{2}^{\rho} \cdot \boldsymbol{e} d V\right) \\
& -\kappa(\rho)\left(f_{\omega_{B}} \boldsymbol{\delta}_{1}^{\rho} \cdot \boldsymbol{e} d V-f_{\omega_{A}} \boldsymbol{\delta}_{1}^{\rho} \cdot \boldsymbol{e} d V\right)\left(f_{\omega_{B}} \boldsymbol{p}_{2} \cdot \boldsymbol{e} d V-f_{\omega_{A}} \boldsymbol{p}_{2} \cdot \boldsymbol{e} d V\right) \\
& -\kappa(\rho)\left(f_{\omega_{B}} \boldsymbol{\delta}_{1}^{\rho} \cdot \boldsymbol{e} d V-f_{\omega_{A}} \boldsymbol{\delta}_{1}^{\rho} \cdot \boldsymbol{e} d V\right)\left(f_{\omega_{B}} \boldsymbol{q}_{2}^{\rho} \cdot \boldsymbol{e} d V-f_{\omega_{A}} \boldsymbol{q}_{2}^{\rho} \cdot \boldsymbol{e} d V\right) \\
& -\kappa(\rho)\left(f_{\omega_{B}} \boldsymbol{\delta}_{1}^{\rho} \cdot \boldsymbol{e} d V-f_{\omega_{A}} \boldsymbol{\delta}_{1}^{\rho} \cdot \boldsymbol{e} d V\right)\left(f_{\omega_{B}} \boldsymbol{\eta}_{2}^{\rho} \cdot \boldsymbol{e} d V-f_{\omega_{A}} \boldsymbol{\eta}_{2}^{\rho} \cdot \boldsymbol{e} d V\right) .
\end{aligned}
$$

We now estimate those 9 terms in the above right hand side. Most of them are evaluated by rescaling and by using (4.4) and (4.15). Each leading term will be part of the topological derivative and will contribute to the scaling term $s(\rho)$.

$1^{\text {st }}$ term. We perform Taylor expansions around $\boldsymbol{x}_{0}$ and $\boldsymbol{x}_{0}+\ell \boldsymbol{e}$ with integral remainder terms like in the proof of Proposition 2 and with the same notations. For the adjoint state $\boldsymbol{p}_{2}$, the integral remainders are denoted by $\boldsymbol{R}_{p_{2}}^{A}$ and $\boldsymbol{R}_{p_{2}}^{B}$. Recall that these remainders are of order $\rho^{2}$ for the $L^{\infty}$ 
norm in $\omega_{A}$ and $\omega_{B}$. Using the notations from (3.9) we obtain

$$
\begin{aligned}
& \left(\kappa(\rho)\left(f_{\omega_{B}}\left(\boldsymbol{u}_{2}-\boldsymbol{u}_{1}\right) \cdot \boldsymbol{e} d V-f_{\omega_{A}}\left(\boldsymbol{u}_{2}-\boldsymbol{u}_{1}\right) \cdot \boldsymbol{e} d V\right)+\Phi(\rho)\right)\left(f_{\omega_{B}} \boldsymbol{p}_{2} \cdot \boldsymbol{e} d V-f_{\omega_{A}} \boldsymbol{p}_{2} \cdot \boldsymbol{e} d V\right) \\
= & \left(\kappa(\rho)\left(\boldsymbol{U}_{2}-\boldsymbol{U}_{1}\right) \cdot \boldsymbol{e}+\Phi(\rho)\right) \boldsymbol{P}_{2} \cdot \boldsymbol{e}+\kappa(\rho)\left(f_{\omega_{B}}\left(\boldsymbol{R}_{u_{2}}^{B}-\boldsymbol{R}_{u_{1}}^{B}\right) \cdot \boldsymbol{e} d V-f_{\omega_{A}}\left(\boldsymbol{R}_{u_{2}}^{A}-\boldsymbol{R}_{u_{1}}^{A}\right) \cdot \boldsymbol{e} d V\right) \boldsymbol{P}_{2} \cdot \boldsymbol{e} \\
& +\left(\kappa(\rho)\left(\boldsymbol{U}_{2}-\boldsymbol{U}_{1}\right) \cdot \boldsymbol{e}+\Phi(\rho)\right)\left(f_{\omega_{B}} \boldsymbol{R}_{p_{2}}^{B} \cdot \boldsymbol{e} d V-f_{\omega_{A}} \boldsymbol{R}_{p_{2}}^{A} \cdot \boldsymbol{e} d V\right) \\
& +\kappa(\rho)\left(f_{\omega_{B}}\left(\boldsymbol{R}_{u_{2}}^{B}-\boldsymbol{R}_{u_{1}}^{B}\right) \cdot \boldsymbol{e} d V-f_{\omega_{A}}\left(\boldsymbol{R}_{u_{2}}^{A}-\boldsymbol{R}_{u_{1}}^{A}\right) \cdot \boldsymbol{e} d V\right)\left(f_{\omega_{B}} \boldsymbol{R}_{p_{2}}^{B} \cdot \boldsymbol{e} d V-f_{\omega_{A}} \boldsymbol{R}_{p_{2}}^{A} \cdot \boldsymbol{e} d V\right) \\
= & \left(\kappa(\rho)\left(\boldsymbol{U}_{2}-\boldsymbol{U}_{1}\right) \cdot \boldsymbol{e}+\Phi(\rho)\right) \boldsymbol{P}_{2} \cdot \boldsymbol{e}+\mathcal{O}\left(\rho^{k+2}\right) .
\end{aligned}
$$

$2^{\text {nd }}$ term. Likewise, by Taylor expansion the second term is

$$
\begin{aligned}
& \left(\kappa(\rho)\left(f_{\omega_{B}}\left(\boldsymbol{u}_{2}-\boldsymbol{u}_{1}\right) \cdot \boldsymbol{e} d V-f_{\omega_{A}}\left(\boldsymbol{u}_{2}-\boldsymbol{u}_{1}\right) \cdot \boldsymbol{e} d V\right)+\Phi(\rho)\right)\left(f_{\omega_{B}} \boldsymbol{q}_{2}^{\rho} \cdot \boldsymbol{e} d V-f_{\omega_{A}} \boldsymbol{q}_{2}^{\rho} \cdot \boldsymbol{e} d V\right) \\
= & \left(\kappa(\rho)\left(\boldsymbol{U}_{2}-\boldsymbol{U}_{1}+f_{\omega_{B}}\left(\boldsymbol{R}_{u_{2}}^{B}-\boldsymbol{R}_{u_{1}}^{B}\right) d V-f_{\omega_{A}}\left(\boldsymbol{R}_{u_{2}}^{A}-\boldsymbol{R}_{u_{1}}^{A}\right) d V\right) \cdot \boldsymbol{e}+\Phi(\rho)\right) f_{\omega}\left(\boldsymbol{q}_{b, 2}-\boldsymbol{q}_{a, 2}\right) \cdot \boldsymbol{e} d V \\
= & -\frac{\frac{\kappa(\rho)}{\rho|\omega|}\left(\frac{1}{\tau_{A}}+\frac{1}{\tau_{B}}\right)}{1+\frac{\kappa(\rho)}{\rho|\omega|}\left(\frac{1}{\tau_{A}}+\frac{1}{\tau_{B}}\right)}\left(\kappa(\rho)\left(\boldsymbol{U}_{2}-\boldsymbol{U}_{1}\right) \cdot \boldsymbol{e}+\Phi(\rho)\right) \boldsymbol{P}_{2} \cdot \boldsymbol{e}+\mathcal{O}\left(\rho^{2 k+1}\right) .
\end{aligned}
$$

$3^{\text {rd }}$ term. The occurrence of $\boldsymbol{\eta}_{2}^{\rho}$ indicates that the third term should be a remainder term. From the variational formulation of (4.24), with $\boldsymbol{\eta}_{2}^{\rho}$ as test function, we obtain

$$
\left|f_{\omega_{B}} \boldsymbol{\eta}_{2}^{\rho} \cdot \boldsymbol{e} d V-f_{\omega_{A}} \boldsymbol{\eta}_{2}^{\rho} \cdot \boldsymbol{e} d V\right| \leq C \kappa(\rho)^{-1 / 2} \rho\left|\xi_{p_{2}}(\rho)\right| .
$$

The third term is thus bounded by

$$
\begin{aligned}
& \left|\kappa(\rho)\left(f_{\omega_{B}}\left(\boldsymbol{u}_{2}-\boldsymbol{u}_{1}\right) \cdot \boldsymbol{e} d V-f_{\omega_{A}}\left(\boldsymbol{u}_{2}-\boldsymbol{u}_{1}\right) \cdot \boldsymbol{e} d V\right)+\Phi(\rho)\right|\left|f_{\omega_{B}} \boldsymbol{\eta}_{2}^{\rho} \cdot \boldsymbol{e} d V-f_{\omega_{A}} \boldsymbol{\eta}_{2}^{\rho} \cdot \boldsymbol{e} d V\right| \\
& \leq C \kappa(\rho)^{1 / 2} \rho\left|\xi_{p_{2}}(\rho)\right|+C \Phi(\rho) \kappa(\rho)^{-1 / 2} \rho\left|\xi_{p_{2}}(\rho)\right| \leq C \rho^{3 k / 2} .
\end{aligned}
$$

$4^{\text {th }}$ term. Again, by Taylor expansion

$$
\begin{aligned}
& -\kappa(\rho)\left(f_{\omega_{B}} \boldsymbol{v}_{1}^{\rho} \cdot \boldsymbol{e} d V-f_{\omega_{A}} \boldsymbol{v}_{1}^{\rho} \cdot \boldsymbol{e} d V\right)\left(f_{\omega_{B}} \boldsymbol{p}_{2} \cdot \boldsymbol{e} d V-f_{\omega_{A}} \boldsymbol{p}_{2} \cdot \boldsymbol{e} d V\right) \\
= & -\kappa(\rho) f_{\omega}\left(\boldsymbol{v}_{b, 1}-\boldsymbol{v}_{a, 1}\right) \cdot \boldsymbol{e} d V\left(\boldsymbol{P}_{2} \cdot \boldsymbol{e}+f_{\omega_{B}} \boldsymbol{R}_{p_{2}}^{B} \cdot \boldsymbol{e} d V-f_{\omega_{A}} \boldsymbol{R}_{p_{2}}^{A} \cdot \boldsymbol{e} d V\right) \\
= & \frac{\kappa(\rho) \Phi(\rho)}{\rho|\omega|}\left(\frac{1}{\tau_{A}}+\frac{1}{\tau_{B}}\right) \boldsymbol{P}_{2} \cdot \boldsymbol{e}+\mathcal{O}\left(\rho^{2 k+1}\right) .
\end{aligned}
$$


$5^{\text {th }}$ term. By a simple rescaling

$$
\begin{aligned}
& -\kappa(\rho)\left(f_{\omega_{B}} \boldsymbol{v}_{1}^{\rho} \cdot \boldsymbol{e} d V-f_{\omega_{A}} \boldsymbol{v}_{1}^{\rho} \cdot \boldsymbol{e} d V\right)\left(f_{\omega_{B}} \boldsymbol{q}_{2}^{\rho} \cdot \boldsymbol{e} d V-f_{\omega_{A}} \boldsymbol{q}_{2}^{\rho} \cdot \boldsymbol{e} d V\right) \\
= & -\kappa(\rho) f_{\omega}\left(\boldsymbol{v}_{b, 1}-\boldsymbol{v}_{a, 1}\right) \cdot \boldsymbol{e} d V f_{\omega}\left(\boldsymbol{q}_{b, 2}-\boldsymbol{q}_{a, 2}\right) \cdot \boldsymbol{e} d V \\
= & -\kappa(\rho) \frac{\Phi(\rho)}{\rho|\omega|}\left(\frac{1}{\tau_{A}}+\frac{1}{\tau_{B}}\right) \frac{\frac{\kappa(\rho)}{\rho|\omega|}\left(\frac{1}{\tau_{A}}+\frac{1}{\tau_{B}}\right)}{1+\frac{\kappa(\rho)}{\rho|\omega|}\left(\frac{1}{\tau_{A}}+\frac{1}{\tau_{B}}\right)} \boldsymbol{P}_{2} \cdot \boldsymbol{e} .
\end{aligned}
$$

$6^{\text {th }}$ term. Like for the third term, the occurence of $\boldsymbol{\eta}_{2}^{\rho}$ indicates that it should be a remainder term. Using the estimate (4.25), it follows

$$
\begin{aligned}
& \kappa(\rho)\left|f_{\omega_{B}} \boldsymbol{v}_{1}^{\rho} \cdot \boldsymbol{e} d V-f_{\omega_{A}} \boldsymbol{v}_{1}^{\rho} \cdot \boldsymbol{e} d V\right|\left|f_{\omega_{B}} \boldsymbol{\eta}_{2}^{\rho} \cdot \boldsymbol{e} d V-f_{\omega_{A}} \boldsymbol{\eta}_{2}^{\rho} \cdot \boldsymbol{e} d V\right| \\
& \leq C \frac{\kappa(\rho)^{1 / 2} \Phi(\rho)}{\rho} \kappa(\rho) \leq C \rho^{5 k / 2-1} .
\end{aligned}
$$

$7^{\text {th }}$ term. Since it involves $\boldsymbol{\delta}_{1}^{\rho}$, it is a remainder term. Using (4.21) yields

$$
\begin{aligned}
& \kappa(\rho)\left|f_{\omega_{B}} \boldsymbol{\delta}_{1}^{\rho} \cdot \boldsymbol{e} d V-f_{\omega_{A}} \boldsymbol{\delta}_{1}^{\rho} \cdot \boldsymbol{e} d V\right|\left|f_{\omega_{B}} \boldsymbol{p}_{2} \cdot \boldsymbol{e} d V-f_{\omega_{A}} \boldsymbol{p}_{2} \cdot \boldsymbol{e} d V\right| \\
& \leq C \kappa(\rho) \Phi(\rho)+C \kappa(\rho) \Phi(\rho) \rho^{2} \leq C \rho^{2 k} .
\end{aligned}
$$

$8^{\text {th }}$ term. Similarly to the seventh term

$$
\kappa(\rho)\left|f_{\omega_{B}} \boldsymbol{\delta}_{1}^{\rho} \cdot \boldsymbol{e} d V-f_{\omega_{A}} \boldsymbol{\delta}_{1}^{\rho} \cdot \boldsymbol{e} d V\right|\left|f_{\omega_{B}} \boldsymbol{q}_{2}^{\rho} \cdot \boldsymbol{e} d V-f_{\omega_{A}} \boldsymbol{q}_{2}^{\rho} \cdot \boldsymbol{e} d V\right| \leq C \rho^{3 k-1} .
$$

$9^{\text {th }}$ term. This last term is a remainder term which, by (4.21) and (4.25), is bounded by

$$
\begin{aligned}
& \kappa(\rho)\left|f_{\omega_{B}} \boldsymbol{\delta}_{1}^{\rho} \cdot \boldsymbol{e} d V-f_{\omega_{A}} \boldsymbol{\delta}_{1}^{\rho} \cdot \boldsymbol{e} d V\right|\left|f_{\omega_{B}} \boldsymbol{\eta}_{2}^{\rho} \cdot \boldsymbol{e} d V-{\underset{\omega}{\omega_{A}}}_{\boldsymbol{f}} \boldsymbol{\eta}_{2}^{\rho} \cdot \boldsymbol{e} d V\right| \\
& \leq C \kappa(\rho) \Phi(\rho) \kappa(\rho)^{-1 / 2} \rho\left|\xi_{u_{2}}(\rho)\right| \leq C \rho^{5 k / 2} .
\end{aligned}
$$

Finally, all leading terms are gathered and give

$$
\left(\frac{1}{\kappa(\rho)}+\frac{1}{\rho|\omega|\left(\frac{1}{\tau_{A}}+\frac{1}{\tau_{B}}\right)}\right)^{-1}\left(\boldsymbol{U}_{2}-\boldsymbol{U}_{1}\right) \cdot \boldsymbol{e} \boldsymbol{P}_{2} \cdot \boldsymbol{e}+\Phi(\rho) \boldsymbol{P}_{2} \cdot \boldsymbol{e},
$$

which, using again the scaling laws (3.2), is equal to

$$
\rho^{k}\left(K\left(\boldsymbol{U}_{2}-\boldsymbol{U}_{1}\right) \cdot \boldsymbol{e}+Q\right) \boldsymbol{P}_{2} \cdot \boldsymbol{e}+\mathcal{O}\left(\rho^{2 k-1}\right) .
$$

All remainder terms are gathered and we keep only the largest terms in $\rho$, which are

$$
\mathcal{O}\left(\rho^{k+2}\right)+\mathcal{O}\left(\rho^{3 k / 2}\right)+\mathcal{O}\left(\rho^{2 k-1}\right) .
$$

Deciding which term is the largest one yields the three regimes for the error term in (3.10). 


\section{Optimization methods}

\subsection{A coupled optimization problem}

The topological derivative provides the optimal location and orientation for a new small bolt. It is therefore a useful tool to decide if it is beneficial to increase the number of bolts in a constrained optimization process for a mechanical system. It is even more useful when coupled to a topology optimization method for the structure. Therefore, we propose a coupled optimization approach between the topology of a structure and the location and number of its bolt connections. Both design variables, namely the structural shape and the bolt locations, are optimized with a gradient-based method. The structure is optimized with the level-set method as presented in Section 5.2, in the spirit of [2]. The optimization of bolt locations is performed with a parametric gradient-based algorithm as explained in Section 5.3.

\subsection{Shape optimization}

\subsubsection{Level-set method}

In this paper, structures are represented with the level-set method as introduced by [20] (see also the textbooks $[19,27]$ ). It offers a large flexibility in topological changes and the boundaries of the structure are explicitly defined. Consider a working domain $\mathcal{D} \subset \mathbb{R}^{3}$ that contains all admissible shapes $\Omega$. A shape $\Omega$ is parameterized by a function $\psi$ defined on $\mathcal{D}$ such that

$$
\left\{\begin{array}{l}
\psi(\boldsymbol{x})=0 \Longleftrightarrow \boldsymbol{x} \in \partial \Omega \cap \mathcal{D}, \\
\psi(\boldsymbol{x})<0 \Longleftrightarrow \boldsymbol{x} \in \Omega, \\
\psi(\boldsymbol{x})>0 \Longleftrightarrow \boldsymbol{x} \in(\mathcal{D} \backslash \bar{\Omega}) .
\end{array}\right.
$$

The shape boundary is evolved in time with the following Hamilton-Jacobi equation

$$
\left\{\begin{aligned}
\frac{\partial \psi}{\partial t}+V|\nabla \psi| & =0 \text { on }\left[0, \infty\left[\times \mathbb{R}^{3},\right.\right. \\
\psi(t=0, \boldsymbol{x}) & =\psi_{0}(\boldsymbol{x}),
\end{aligned}\right.
$$

where $V(t, \boldsymbol{x})$ is the normal velocity field. Equation (5.1) is solved by linearization as a transport equation with the method of characteristics (see [9] and the corresponding free software [10]). This level-set approach is complemented with the ersatz material approach which replaces void by a weak artificial material, thus avoiding singularity issues when solving the elasticity problem in the full computational domain $\mathcal{D}$.

\subsubsection{Hadamard's boundary variation method}

Hadamard's boundary variation method is used to compute the velocity field $V$ of (5.1). Introduced by [16], this method defines a notion of differentiation with respect to the position of a shape $\Omega$ [21,30]. Let $\Omega \subset \mathbb{R}^{3}$ be a given reference shape, assumed to be a smooth and bounded open set. Let $\boldsymbol{\theta} \in W^{1, \infty}\left(\mathbb{R}^{3}, \mathbb{R}^{3}\right)$ be a displacement field. The idea of Hadamard's boundary variation method is to transport the reference domain $\Omega$ into an admissible shape $\Omega_{\theta}=(\boldsymbol{I} \boldsymbol{d}+\boldsymbol{\theta})(\Omega)$. In other words, the vector field $\boldsymbol{\theta}$ moves all points of $\Omega$ from a location $\boldsymbol{x}$ to a deformed location $\boldsymbol{x}+\boldsymbol{\theta}(\boldsymbol{x})$.

Definition 2. Let $J(\Omega)$ be a function from the set of admissible shapes to $\mathbb{R}$. The function $J(\Omega)$ is said to be shape differentiable if there exists a continuous linear form $J^{\prime}(\Omega)$ acting on $W^{1, \infty}\left(\mathbb{R}^{3}, \mathbb{R}^{3}\right)$ such that

$$
J((\boldsymbol{I} \boldsymbol{d}+\boldsymbol{\theta})(\Omega))=J(\Omega)+J^{\prime}(\Omega)(\boldsymbol{\theta})+o(\boldsymbol{\theta}), \text { where } \lim _{\boldsymbol{\theta} \rightarrow \mathbf{0}} \frac{|o(\boldsymbol{\theta})|}{\|\boldsymbol{\theta}\|_{W^{1, \infty}\left(\mathbb{R}^{3}, \mathbb{R}^{3}\right)}}=0 .
$$


The function $J^{\prime}(\Omega)$ is called the shape derivative of the shape functional $J$. We give a basic example of shape derivatives which is useful for the sequel (for a proof, see e.g. [1]).

Proposition 4. Let $\Omega$ be a smooth, bounded, open set of $\mathbb{R}^{3}$. Let $\boldsymbol{u}$ be the solution of the elasticity system (2.2). Define a shape functional $J$, called the compliance, by

$$
J(\Omega)=\int_{\Gamma_{N}} \boldsymbol{g} \cdot \boldsymbol{u} d S=\int_{\Omega} \mathrm{A} \varepsilon(\boldsymbol{u}): \varepsilon(\boldsymbol{u}) d V .
$$

Then, $J$ is shape differentiable and, for any $\boldsymbol{\theta} \in W^{1, \infty}\left(\mathbb{R}^{3}, \mathbb{R}^{3}\right)$ such that $\boldsymbol{\theta}=0$ on $\Gamma_{N} \cup \Gamma_{D}$, its directional derivative is

$$
J^{\prime}(\Omega)(\boldsymbol{\theta})=-\int_{\Gamma} \mathrm{A} \varepsilon(\boldsymbol{u}): \varepsilon(\boldsymbol{u}) \boldsymbol{\theta} \cdot \boldsymbol{n} d S,
$$

where $\boldsymbol{n}$ is the outward normal to $\Omega$.

\subsection{Location optimization}

The bolt has a fixed shape, length and orientation. The only design-variable of the bolt is the center $\boldsymbol{x}_{0}$ of its head $\omega_{A}$. Bolt location optimization is performed with a parametric gradient-based algorithm

$$
\boldsymbol{x}_{0}^{k+1}=\boldsymbol{x}_{0}^{k}-\delta^{k} \frac{\partial J}{\partial \boldsymbol{x}_{0}}\left(\boldsymbol{x}_{0}^{k}\right),
$$

where $\boldsymbol{x}_{0}^{k}$ is the center of the head of the idealized bolt at iteration $k, \delta^{k}>0$ is the descent step and $\frac{\partial J}{\partial \boldsymbol{x}_{0}}\left(\boldsymbol{x}_{0}^{k}\right)$ is the partial derivative of the objective function with respect to the location $\boldsymbol{x}_{0}$, computed according to Hadamard's boundary variation method. The spheres representing the head and the threads are simply translated (they are rotation invariant). Therefore, the deformation vector $\boldsymbol{\theta}$ is constant on $\omega_{A}$. The following result gives the form of the derivative of the bolt constitutive behavior law and specific mechanical constraints.

Proposition 5. Let $\omega_{x_{0}}$ be a smooth bounded, open set $\omega \subset \mathbb{R}^{3}$ translated so that its center is $\boldsymbol{x}_{0} \in \mathcal{D}$. Let $f \in W^{1,1}\left(\mathbb{R}^{3}, \mathbb{R}^{3}\right)$ and $J$ a function from $\mathcal{D}$ to $\mathbb{R}$ defined by

$$
J\left(\boldsymbol{x}_{0}\right)=f_{\omega_{x_{0}}} f d V=\frac{1}{|\omega|} \int_{\omega_{x_{0}}} f d V .
$$

Then, $J$ is differentiable and it satisfies

$$
\frac{\partial J}{\partial \boldsymbol{x}_{0}}\left(\boldsymbol{x}_{0}\right) \cdot \boldsymbol{\theta}=J^{\prime}\left(\boldsymbol{x}_{0}\right)(\boldsymbol{\theta})=\frac{\boldsymbol{\theta}}{|\omega|} \cdot \int_{\partial \omega_{\boldsymbol{x}_{0}}} f \boldsymbol{n} d S, \quad \forall \boldsymbol{\theta} \in \mathbb{R}^{3} .
$$

where $\boldsymbol{n}$ is the outward normal to $\omega_{\boldsymbol{x}_{0}}$.

Proof. To prove (5.4) we identify the function $J\left(\boldsymbol{x}_{0}\right)$ with a shape functional $J\left(\omega_{\boldsymbol{x}_{0}}\right)$. The classical shape derivative (see [2]) gives, for any $\boldsymbol{\theta} \in W^{1, \infty}\left(\mathbb{R}^{3}, \mathbb{R}^{3}\right)$,

$$
J^{\prime}\left(\omega_{\boldsymbol{x}_{0}}\right)(\boldsymbol{\theta})=\frac{|\omega| \int_{\partial \omega_{\boldsymbol{x}_{0}}} f \boldsymbol{\theta} \cdot \boldsymbol{n} d S-\left(\int_{\omega_{\boldsymbol{x}_{0}}} f d V\right)\left(\int_{\partial \omega_{\boldsymbol{x}_{0}}} \boldsymbol{\theta} \cdot \boldsymbol{n} d S\right)}{|\omega|^{2}} .
$$


Restricting our attention to constant vector fields $\boldsymbol{\theta}$ yields that the directional derivative of the volume is zero, i.e. $\int_{\partial \omega_{x_{0}}} \boldsymbol{\theta} \cdot \boldsymbol{n} d S=0$. Thus we deduce the desired formula by the identification, for constant vectors $\boldsymbol{\theta} \in \mathbb{R}^{3}$,

$$
J^{\prime}\left(\omega_{\boldsymbol{x}_{0}}\right)(\boldsymbol{\theta})=\frac{\partial J}{\partial \boldsymbol{x}_{0}}\left(\boldsymbol{x}_{0}\right) \cdot \boldsymbol{\theta}
$$

\section{$6 \quad$ Numerical illustrations}

Although we derived a topological derivative for a pre-stressed bolt, here for the sake of simplicity, our numerical illustrations are computed without pre-tension. In such a case, the topological derivative is given by Corollary 1 . The reason for not taking into account the pre-stress here is that it usually induces some inter-penetration of the structure if one does not use a contact model between the two parts connected by the bolt. Since a contact model add some complexity to the computations, we prefer not to use it in a first step of assessment and, as a consequence, we ignore the pre-stress in the bolt, which thus behaves as a spring. Therefore, in all examples below, the interface $\Gamma_{c}$ is a crack without contact conditions. The interested reader is referred to [24] for applications of our coupled optimization of both structure and connections with a pre-tensioned bolt and a sliding contact model. All our numerical results are obtained with the finite element software FreeFem $++[17]$.

\section{$6.12 \mathrm{~d}$ use case}

The present paper gives a complete derivation of the topological derivative in $3 \mathrm{~d}$ but, as already said in Remark 7, the same result (Corollary 1) holds true in 2d (see formula (3.12) and [22] Chapter 5 , Section 5.2, for details).

\subsubsection{Description of the problem}

The following academic, but meaningful, $2 \mathrm{~d}$ use case illustrates the coupled optimization with a small spring (without pre-tension). The working domain is a plate of 2 units long and 1 unit wide with an opening gap through its center as displayed in Fig. 5. The mesh contains 69420 triangular elements with a minimal and maximal size of $3.3 \times 10^{-3}$ and $1.2 \times 10^{-2}$. The Young's modulus is taken equal to 10 and the Poisson's coefficient is 0.3 . The length of the spring is $\ell=0.3$ and its stiffness is $K=5$. Once a spring is inserted, its size is $\rho=2.5 \times 10^{-2}$. All material properties are adimensional and can be changed as the user wants. The plate is clamped on the bottom and loaded on its upper boundary by a sinusoidal force $\boldsymbol{g}=\left(0, g_{y}\right)$, with

$$
g_{y}= \begin{cases}-\cos (\pi x) & \text { for } 0.5 \leq x \leq 1.5 \\ -\frac{3}{4} \cos (\pi x) & \text { for } 1.5 \leq x \leq 2\end{cases}
$$

This use case, which considers a single part with an opening gap, perfectly fits our framework for using the topological derivative in order to decide where to put a spring. Actually, the role of the spring is to close the gap. Moreover, as already said in Remark 1, the asymptotic analysis is valid regardless of the condition on the interface. Therefore, homogeneous Neumann boundary condition is applied on the opening gap. Of course, this may lead to some interpenetration which could be corrected by taking into account a contact model (see [24] for more details).

The topology of the plate is initialized with holes (see Fig. 5b) for the purpose of further topology optimization. The initial compliance is then $J_{0}(\Omega)=0.3210$. 


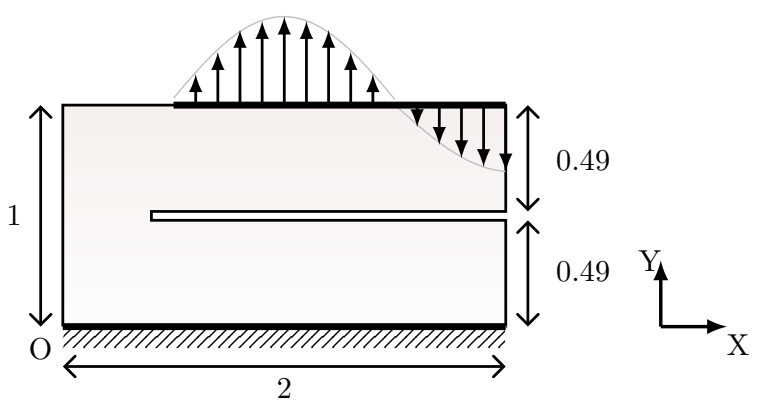

(a)

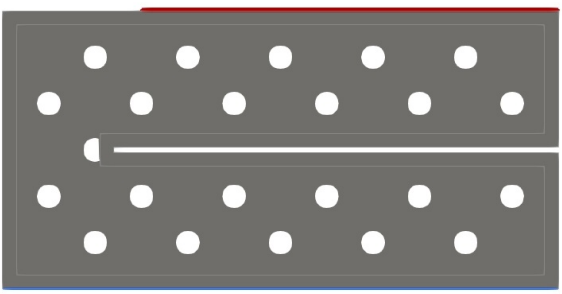

(b)

Figure 5: Load case (a) and the initial topology of the structure (b)

\subsubsection{Test of the topological derivative}

The problem is to place a small spring in order to decrease the compliance $J(\Omega)=\int_{\Gamma_{N}} \boldsymbol{g} \cdot \boldsymbol{u} d S$. Recall that the topological derivative $D J\left(\boldsymbol{x}_{0}, \boldsymbol{e}\right)$ is the same for all values $k>0$ of the scaling parameter for the spring stiffness in $2 \mathrm{~d}$, see (3.12). The topological derivative is evaluated to search for both the optimal location $\boldsymbol{x}_{0}$ and orientation $\boldsymbol{e}$. We define a design-space for which both spheres, $\omega_{A}$ and $\omega_{B}$, are entirely inside the structure. Fig. 6a displays the cartography of the topological derivative where the non-design space is darkened. The minimum of $D J$ is obtained at the point $\boldsymbol{x}_{0}=(1.53,0.43)$ and for a direction oriented at $\varphi=7 \pi / 12 \mathrm{rad}$ (or $105^{\circ}$ with respect to the horizontal axis) as shown in Fig. 6 b.

To test the accuracy of the prediction of the topological derivative, we include the spring model in the structure, for its given size $\rho$ and for some choice of the scaling exponent $k>0$, and we compte the resulting compliance $J_{\rho}(\Omega)$. To be more realistic, a surrounding of material is added around each inclusion $\omega_{A}$ and $\omega_{B}$. This non-design domain is spherical-shaped and twice the size of the inclusion. Its role is to ensure the correct transmission of efforts into the structure. It is the slight extra material that modifies the hole next to each inclusion (see Fig. 6b). This non-design domain is not taken into account by the asymptotic analysis. It is added in the structure after putting the spring indicated by the topological derivative. Nevertheless, the impact of this extra material is barely distinguishable.

Although the scaling exponent $k>0$ does not play any role in the formula of the topological derivative $D J\left(\boldsymbol{x}_{0}, \boldsymbol{e}\right)$, it does so in the compliance $J_{\rho}(\Omega)$ of the resulting structure, including the spring. Therefore, it is worth exploring its influence and results are gathered in Table 1. As could be expected, the most efficient spring corresponds to the smallest value $k=0.5$, which brings an improvement of about $17 \%$ of the compliance. Clearly, there is a loss of efficiency for $k=2$ and $k=4$. Consequently, the scalings $k=2$ and $k=4$ will not be taken into account in the following.

\begin{tabular}{|c|c|c|c|c|}
\cline { 2 - 5 } \multicolumn{1}{c|}{} & $D J\left(\boldsymbol{x}_{0}, \boldsymbol{e}\right)$ & $\boldsymbol{x}_{0}$ & $\varphi(\mathrm{rad})$ & $J_{\rho}(\Omega)$ \\
\hline$k=0.5$ & -5.648 & $(1.53,0.43)$ & $\frac{7 \pi}{12}$ & 0.2674 \\
\hline$k=1$ & -5.648 & $(1.53,0.43)$ & $\frac{7 \pi}{12}$ & 0.2803 \\
\hline$k=2$ & -5.648 & $(1.53,0.43)$ & $\frac{7 \pi}{12}$ & 0.3174 \\
\hline$k=4$ & -5.648 & $(1.53,0.43)$ & $\frac{7 \pi}{12}$ & 0.3207 \\
\hline
\end{tabular}

Table 1: Summary of the topological derivative tests (recall that $J_{0}(\Omega)=0.3210$ ) 


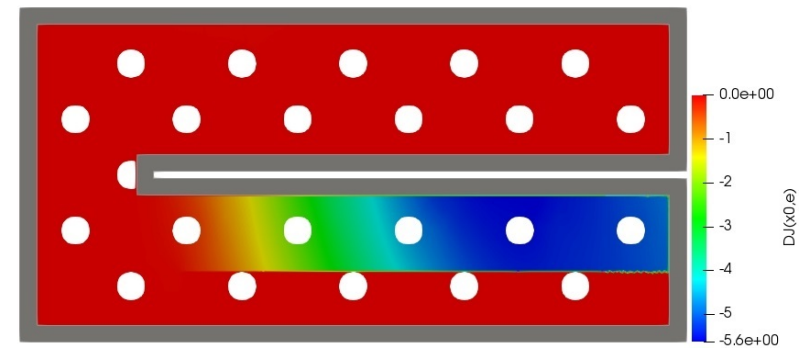

(a)

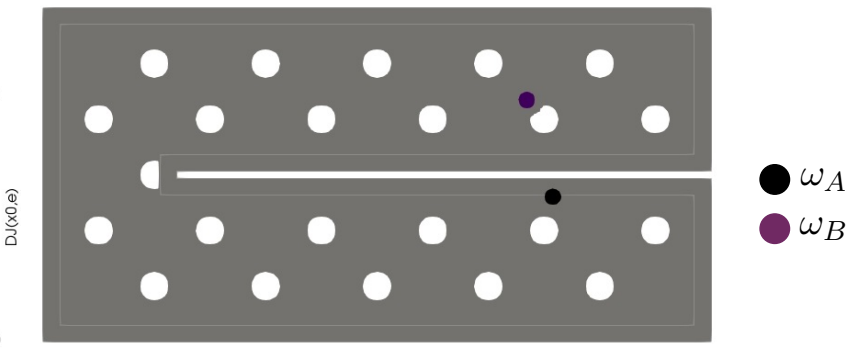

(b)

Figure 6: For $\varphi=\frac{7 \pi}{12} \mathrm{rad}$, cartography of $D J$ (a) and location of the optimal spring at $\boldsymbol{x}_{0}=$ $(1.53,0.43)(b)$

According to this first computation, a first spring with $k=0.5$ is placed at the point $\boldsymbol{x}_{0,1}=$ $(1.53,0.43)$, oriented by the angle $\varphi_{1}=7 \pi / 12 \mathrm{rad}$, as indicated by the topological derivative. For this new configuration, a new map of the topological derivative is computed in order to find where it could be interesting to place a second spring. The cartography of this second topological derivative is displayed in Fig. 7a. Negative values of $D J$, for which $\omega_{B, 2}$ would be partially or totally in the non-design-space, are truncated. They are excluded from the analysis and are displayed in white as well as the holes of the structure. The topological derivative places the second spring at the point $\boldsymbol{x}_{0,2}=(1.80,0.31)$ with the angle $\varphi_{2}=13 \pi / 36 \mathrm{rad}$ as displayed in Fig. 7 . With this second spring, the compliance decreases to $J_{\rho}(\Omega)=0.2231$.

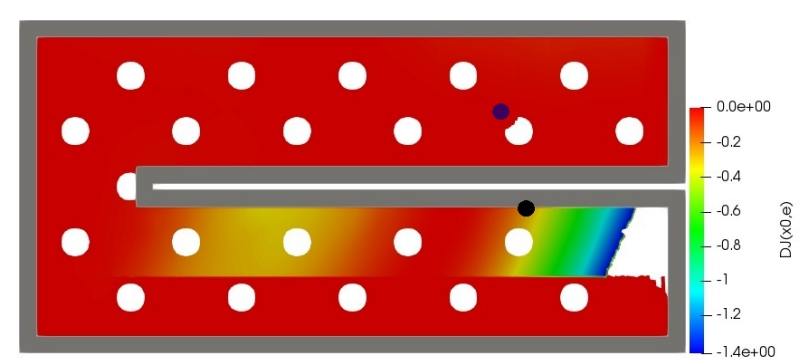

(a)

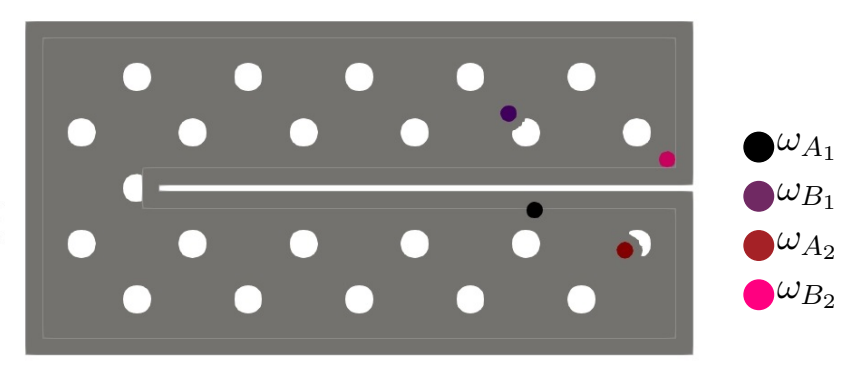

(b)

Figure 7: Cartography of $D J$ for $k=0.5$ to place a second spring (a) and the corresponding optimal configuration (b)

\subsubsection{Coupled optimization of both structure and springs location}

The problem is to minimize the volume under an upper bound constraint on the compliance, equal to 0.35 . The volume $V(\Omega)$ and the compliance $C\left(\Omega, \boldsymbol{x}_{0}\right)$ read

$$
V(\Omega)=\int_{\Omega} d V \text { and } C\left(\Omega, \boldsymbol{x}_{0}\right)=\int_{\Gamma_{N}} \boldsymbol{g} \cdot \boldsymbol{u} d S,
$$

where $\boldsymbol{x}_{0}$ is the location of the spring. This optimization problem is formulated as follows

$$
\min _{\substack{\Omega \in \mathcal{U}_{a d}, x_{0} \in \Omega \\ \text { s.t. } C\left(\Omega, x_{0}\right) \leq 0.35}} V(\Omega),
$$

where $\mathcal{U}_{a d}$ is the set of admissible shapes of the structure. The constraint is taken into account with the augmented Lagrangian functional $J(\Omega)$, so that the problem (6.1) is rewritten as

$$
\min _{\left(\Omega, x_{0}\right)} \max _{\alpha \geq 0}\left\{J\left(\Omega, \boldsymbol{x}_{0}\right)=V(\Omega)+\alpha\left(C\left(\Omega, \boldsymbol{x}_{0}\right)-0.35\right)+\frac{\beta}{2}\left(C\left(\Omega, \boldsymbol{x}_{0}\right)-0.35\right)^{2}\right\},
$$


where $\alpha \geq 0$ and $\beta \geq 0$ are respectively Lagrange multiplier and penalty parameter for the constraint.

\subsubsection{Optimization with one spring}

Two independent computations are presented below. First, we perform a classic topology optimization of the structure with a fixed spring (placed by the initial topological derivative). Second, the optimization of the structure is coupled with the parametric optimization of the spring location.

- Structure optimization with fixed spring

Let us consider the spring given by the topological derivative on the structure initialized with holes (see Fig. 6b). The structure is classically optimized while the spring location is not a design-variable yet. Optimal shapes for $k=0.5$ and $k=1$ are given in Fig. 8 and they share the same topologies. For $k=0.5$, the final volume is $V(\Omega)=0.6436$. For $k=1$, the spring is less stiff and the structure requires more material to fulfil the compliance constraint. Then, the structure is heavier with a final volume of $V(\Omega)=0.6799$.

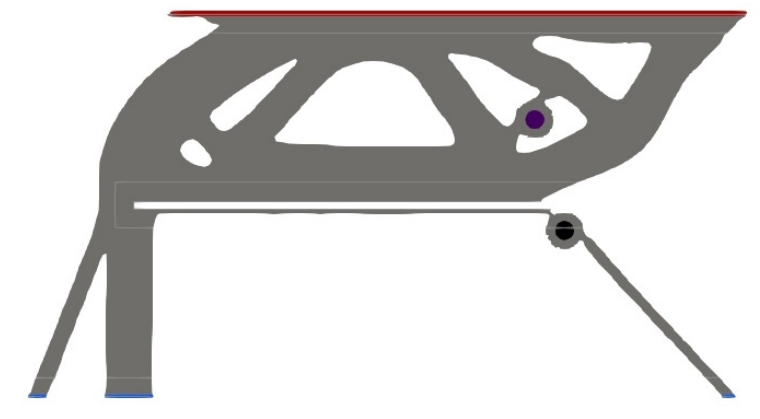

(a) $k=0.5$

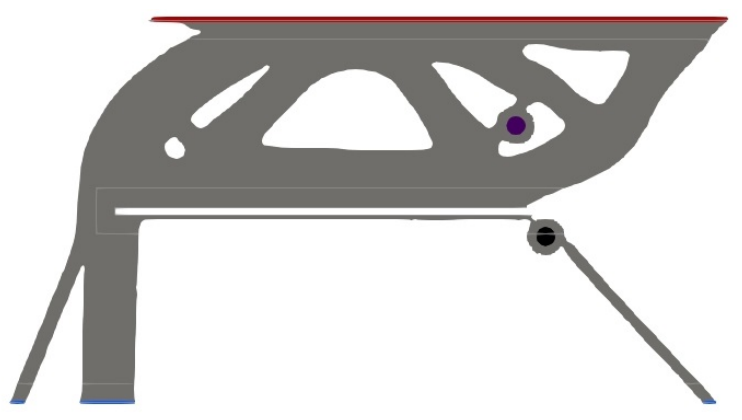

(b) $k=1$

Figure 8: Optimal shapes for $k=0.5$ and $k=1$, for a fixed spring

- Coupled optimization structure / location

Let us consider the spring given by the topological derivative on the structure initialized with holes. Now, the spring location $\boldsymbol{x}_{0}$ is a design-variable, as well as the structure. Optimal shapes for $k=0.5$ and $k=1$ are given in Fig. 9. The case $k=0.5$ gives a completely new topology. The final volume and spring location are $V(\Omega)=0.5460$ and $\boldsymbol{x}_{0}=(0.75,0.42)$. The coupling provides a great improvement of the volume compared to the classical structure optimization. The case $k=1$ leads to a topology very similar to that of Fig. 8 where the spring was fixed. Nevertheless, the coupling brings a better weight reduction with a final volume of $V(\Omega)=0.6275$. Moreover, the spring moves from an initial location at $\boldsymbol{x}_{0}=(1.54,0.39)$ to the final point $\boldsymbol{x}_{0}=(1.34,0.44)$, which corresponds to an amplitude of displacement of 0.21 .

\subsubsection{Optimization with two springs}

This section compares the optimal shapes with two springs resulting from a classic topology optimization and a coupled optimization of both structure and springs locations. Both spring are scaled by $k=0.5$ and are placed and oriented by the topological derivative. The optimal shape at Fig. 10a results from the topology optimization with two fixed springs initially placed and oriented by the topological derivative (see Fig. 7b). The final volume is $V(\Omega)=0.6517$. The left part of the structure is of the same kind as the classic optimal shape with one fixed spring (see Fig. 8a). Springs are connected by means of thin bars. The second spring stiffens the structure and thus allows a better weight reduction than with only the first spring. Unfortunately, this structure is 


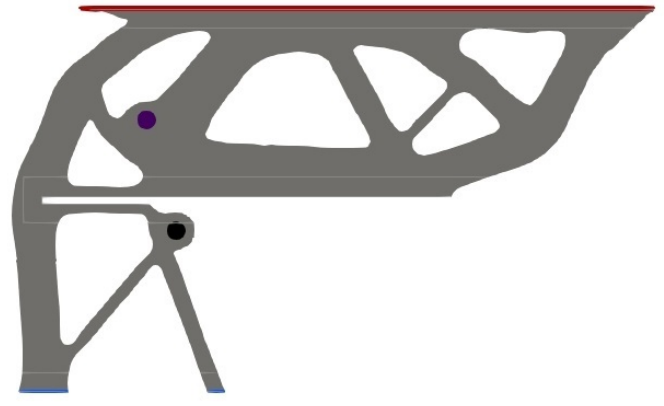

(a) $k=0.5$

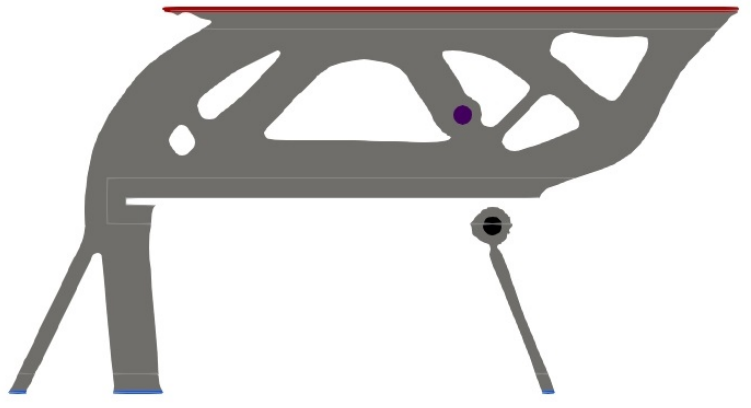

(b) $k=1$

Figure 9: Optimal shapes and spring locations for $k=0.5$ and $k=1$

heavier than the optimal structure with one spring resulting from a coupled optimization (see Fig. 9a).

Fig. 10b shows the optimal shape and springs locations for a coupled optimization of both structure and locations. Final springs locations are $\boldsymbol{x}_{0,1}=(1.18,0.44)$ and $\boldsymbol{x}_{0,2}=(1.79,0.31)$, which correspond to amplitude of displacement of 0.35 for the first spring and 0.01 for the second one. The structure has a different topology and a final volume of $V(\Omega)=0.5564$. This is the best performance obtained. It illustrates well the relevance and the effectiveness of the coupled optimization.

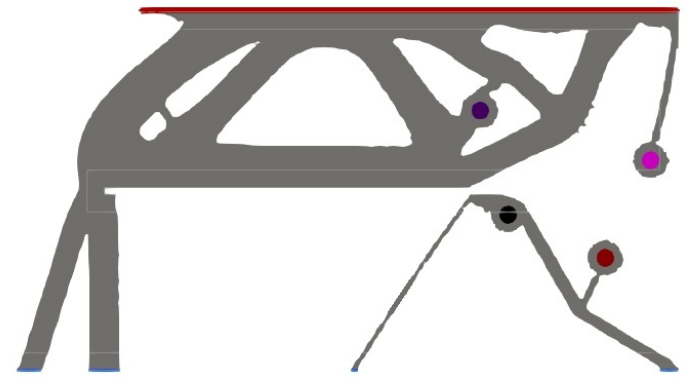

(a)

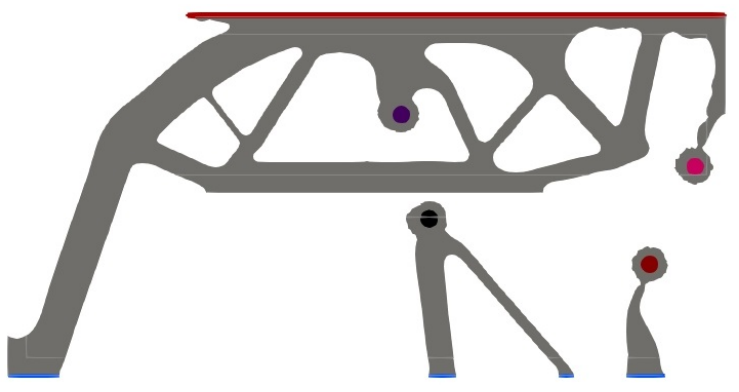

(b)

Figure 10: Optimal shape with two fixed springs (a) and coupled optimization of both structure and springs location (b)

\section{$6.23 d$ use case}

\subsubsection{Description of the problem}

Let us consider two parallelipipedic parts pictured in Fig. 11. They are contiguous but not bounded to each other: in other words, they are separated by a crack. The mesh contains 293864 tetrahedral elements with a minimal and maximal size of $1.2 \times 10-2$ and $5.9 \times 10^{-2}$. The assembly is clamped on the bottom and on the left side. A force $\boldsymbol{g}=\left(0,5 \cos \left(\frac{x}{2}\right), 0\right)$ is applied on the top. Both parts are made of the same material with an adimensional Young's modulus $E=1$ and Poisson's coefficient 0.3 . Springs are characterized by their size $\rho=5 \times 10^{-2}$, length $\ell=5 \times 10^{-1}$ and rigidity $K=2.5$.

The problem is to decrease the compliance $J(\Omega)=\int_{\Gamma_{N}} \boldsymbol{g} \cdot \boldsymbol{u} d S$ of the system by introducing a first then a second small spring with the topological derivative. The initial compliance of the system without spring is $J_{0}(\Omega)=38.06$. No coupling with shape and topology optimization is performed here and the reader is rather referred to our other work [24] for such examples. 


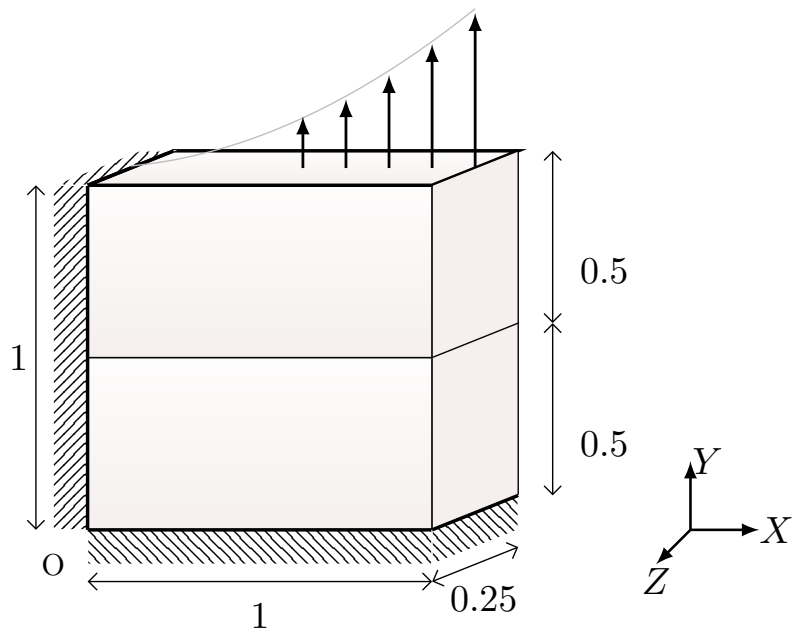

Figure 11: Setting of the 3d use case

Three different uses of the topological derivative are performed with the couple $\left(\boldsymbol{x}_{0}, \boldsymbol{e}\right)$. For graphical purposes, the orientation $\boldsymbol{e}$ is given is spherical coordinates $\boldsymbol{e}=(\sin (\varphi) \cos (\Psi), \sin (\varphi) \sin (\Psi), \cos (\varphi))$. The first test is to search for both the optimal location $\boldsymbol{x}_{0}$ and optimal orientation $\boldsymbol{e}$. The second test consists in fixing a location $\boldsymbol{x}_{0}$ and finding the orientation $\boldsymbol{e}$ which minimizes $D J$. The third test, on the contrary, finds the best location $\boldsymbol{x}_{0}$ for a fixed orientation $\boldsymbol{e}$. The topological derivative is then computed for all candidate point $\boldsymbol{x}_{0}$ to place the first small spring. This last computation is reiterated to search for the best location of a second spring with the same orientation.

\subsubsection{Search for the best location $x_{0}$ and orientation $e$}

This test searches simultaneously for both the optimal location of the spring head $\boldsymbol{x}_{0}$ and the best orientation $e$ to decrease the compliance. The topological derivative indicates that the optimal spring should be placed at the point $\boldsymbol{x}_{0}=(0.90,0.90,0.11)$ with the orientation $(\varphi, \Psi)=\left(\frac{\pi}{2},-\frac{\pi}{2}\right)$. With such a spring, the compliance indeed decreases to $J_{\rho}(\Omega)=24.49$.

\subsubsection{Fix the location $x_{0}$ and search for the best orientation $e$}

Let us fix the location of the center of the spring head at the point $\boldsymbol{x}_{0}=(0.30,0.70,0.125)$. To find the best orientation $\boldsymbol{e}$, the cartography of the topological derivative is plotted on Fig. 12a in terms of the angles $\varphi$ and $\Psi$ from $0^{\circ}$ to $360^{\circ}$ every five degrees. It features two minimum that correspond to the same orientation indeed. This cartography is computationally cheap since it requires only the resolution of one elasticity system (the problem is self-adjoint with the compliance as objective function). Varying the vector $\boldsymbol{e}$ is just some algebraic manipulation in formula (3.11). As a conclusion, the optimal spring has the orientation $(\varphi, \Psi)=\left(\frac{\pi}{2}, \frac{13 \pi}{9}\right)$ as displayed in Fig. 12b. The compliance with this spring is $J_{\rho}(\Omega)=36.78$.

\subsubsection{Fix the orientation $e$ and search for the best location $x_{0}$}

Let us now fix the spring orientation to be vertical. The cartography of the topological derivative to place the first spring is displayed in Fig. 13. The optimal small spring is placed at the point $\boldsymbol{x}_{0,1}=(0.90,0.90,0.11)$. The new compliance is $J_{\rho}(\Omega)=24.49$, which is quite efficient.

Let us place a second spring with a new evaluation of the topological derivative, taking into account the first spring. The cartography of the topological derivative and the resulting second spring are displayed in Fig. 14. The second spring is best located at $\boldsymbol{x}_{0,2}=(0.90,0.63,0.14)$, which overlaps the first one. It may seem quite deceptive to obtain almost the same location but it 


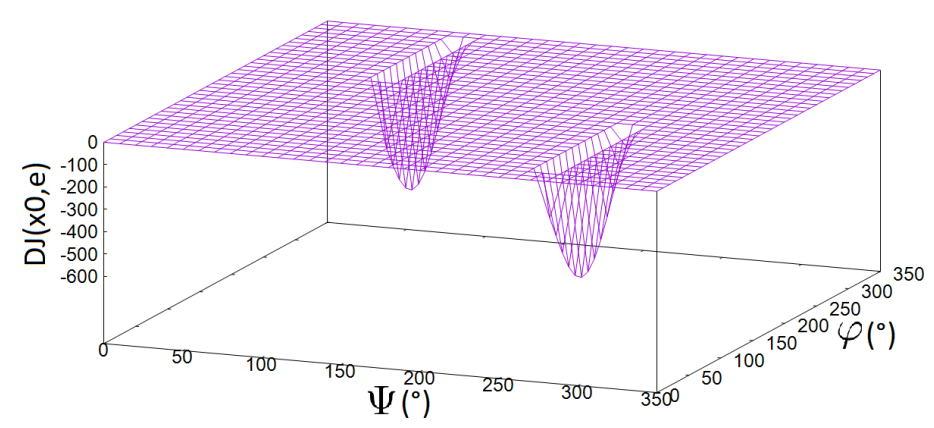

(a)

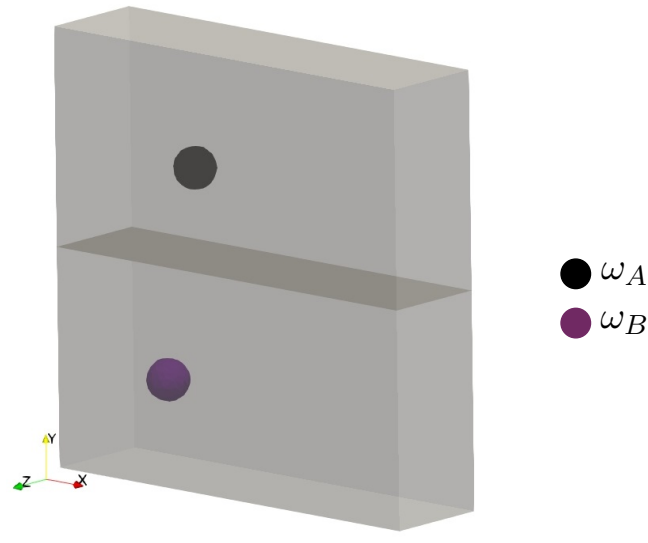

(b)

Figure 12: Cartography of the topological derivative in terms of angles $(\varphi, \Psi)$ for fixed $\boldsymbol{x}_{0}=$ $(0.30,0.70,0.125)$ (a) and the spring at the optimal orientation $(\varphi, \Psi)=\left(\frac{\pi}{2}, \frac{13 \pi}{9}\right)(\mathrm{b})$

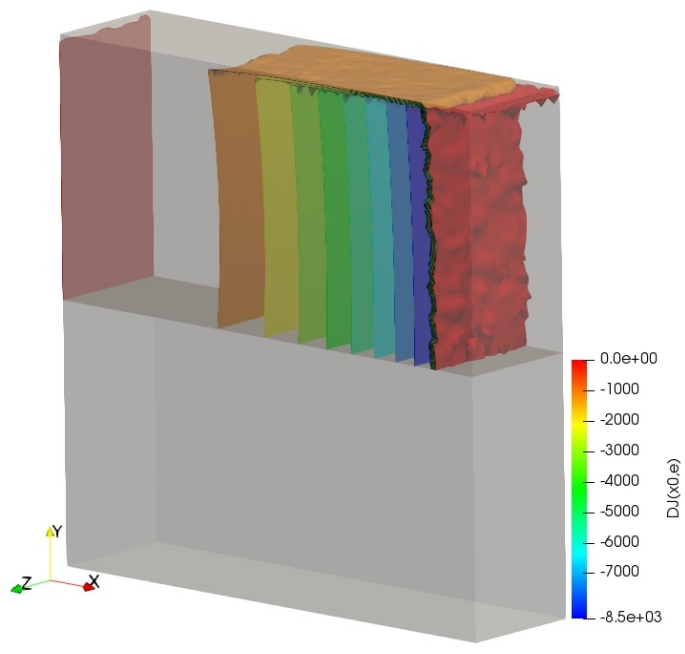

(a)

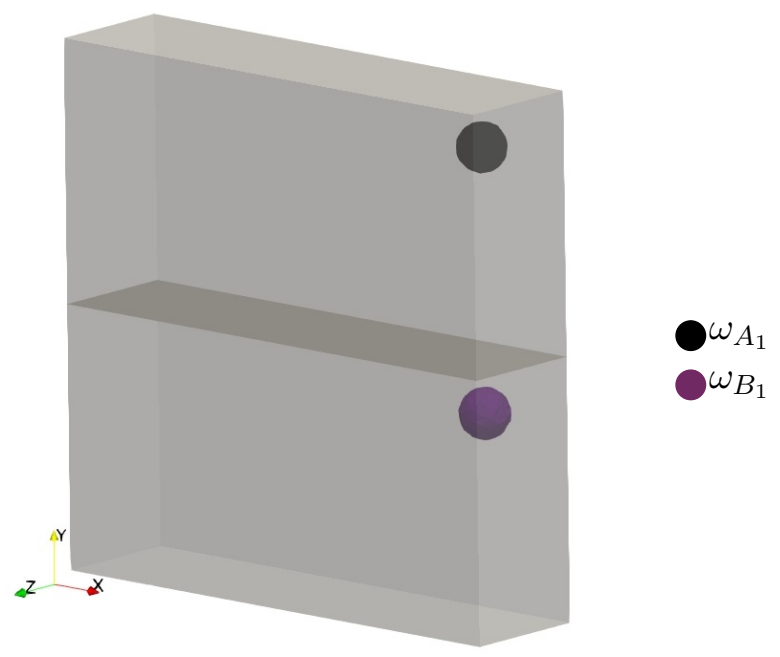

(b)

Figure 13: Cartography of the topological derivative for fixed vertical orientation (a) and the resulting optimal spring (b)

highlights the need of a stronger spring at the same location. The compliance with two springs is $J_{\rho}(\Omega)=19.07$.

To avoid non-physical overlapping of springs, a prescribed minimal distance between springs locations is enforced. It states that springs must be separated by at least four times the radius $\rho$ in the direction $\boldsymbol{X}$. The new cartography and the second optimal spring placed at $\boldsymbol{x}_{0,2}=(0.70,0.83,0.14)$ are displayed in Fig. 15. The new compliance is $J_{\rho}(\Omega)=23.68$. 


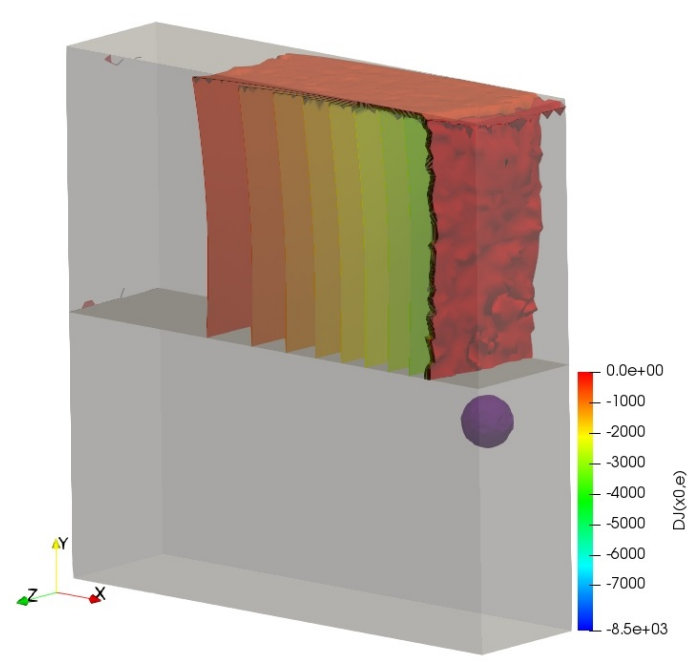

(a)

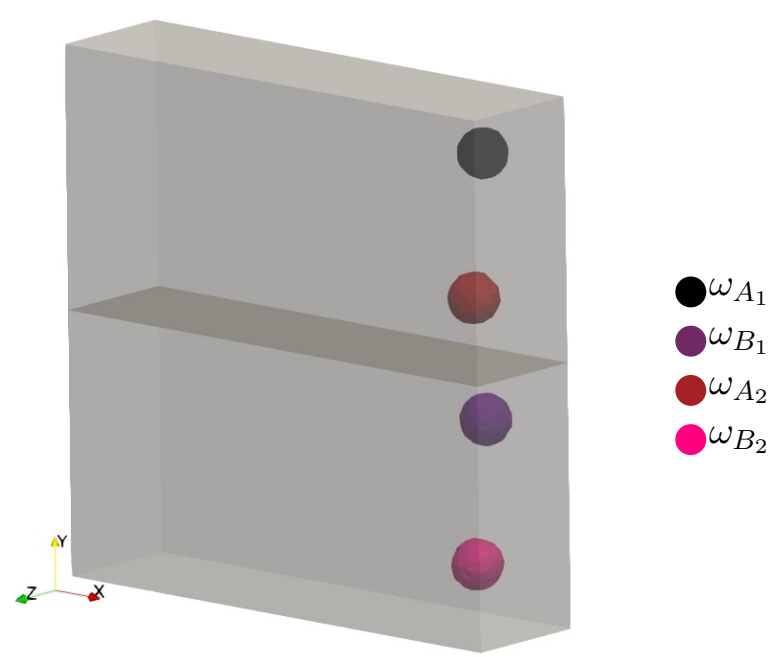

(b)

Figure 14: Cartography of the topological derivative (a) and the resulting second overlapping spring (b)

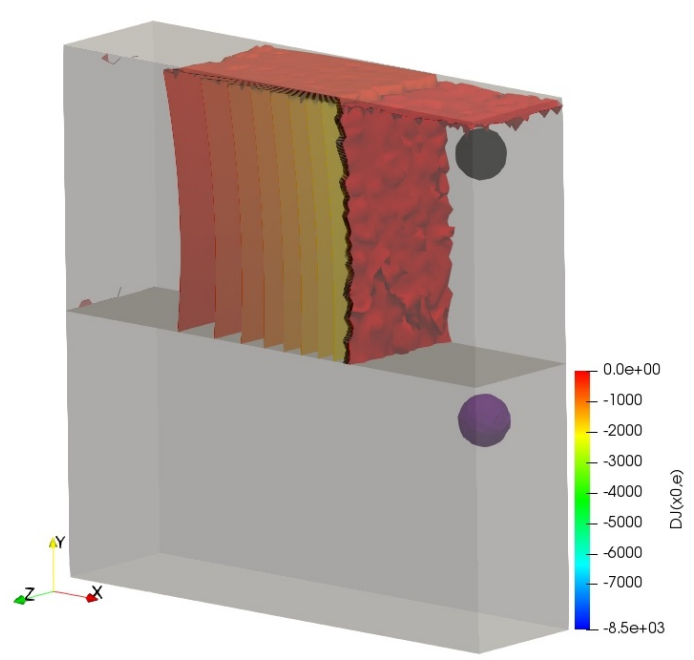

(a)

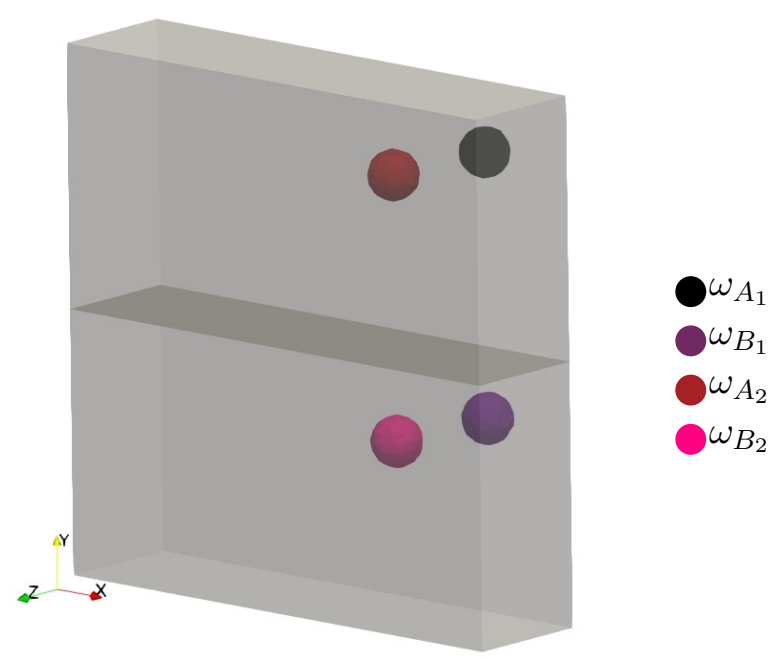

(b)

Figure 15: Cartography of the topological derivative with a non-overlapping condition (a) and the resulting second optimal spring (b) 


\section{Acknowledgements}

This work was supported by the Association Nationale de la Recherche et de la Technologie (ANRT). It was carried out in the context of a CIFRE thesis between Groupe RENAULT, Technocentre and Centre de Mathématiques Appliquées at Ecole Polytechnique (CMAP).

\section{References}

[1] G. Allaire. Conception optimale de structures. Springer-Verlag, Berlin, Mathématiques et Applications 58, 2007.

[2] G. Allaire, F. Jouve, and A. M. Toader. Structural optimization using sensitivity analysis and a level-set method. J. Comp. Phys., 194(1):363-393, 2004.

[3] G. Allaire, F. Jouve, and N. Van Goethem. Damage evolution in brittle materials by shape and topological sensitivity analysis. J. Comp. Phys., 203:5010-5044, 2011.

[4] H. Ammari and H. Kang. Reconstruction of small inhomogeneities from boundary measurements. Springer, Berlin, 2004.

[5] S. Amstutz. The topological asymptotic for the navier-stokes equations. ESAIM: Control, Optimisation and Calculus of Variations, 11:401-425, 2005.

[6] S. Amstutz, I. Horchani, and M. Masmoudi. Crack detection by the topological gradient method. Control and Cybernetics, 34(1):81-101, 2005.

[7] D. Auroux, M. Masmoudi, and L. Belaid. Image restoration and classification by topological asymptotic expansion. Variational formulations in mechanics : theory and applications, pages 23-42, 2006.

[8] D. Bojczuk and A. Rebosz-Kurdek. Optimal design of bar structures with their supports in problems of stability and free vibrations. Journal of theoretical and applied mechanics, $52(2): 533-546,2014$.

[9] C. Bui, C. Dapogny, and P. Frey. An accurate anisotropic adaptation method for solving the level set advection equation. International Journal for Numerical Methods in Fluids, 70(7):899-922, 2012.

[10] C. Bui, C. Dapogny, and P. Frey. Advect. https://github.com/ISCDtoolbox/Advection, 2015 .

[11] J. Céa, S. Garreau, P. Guillaume, and M. Masmoudi. The shape and topological optimizations connection. Comput. Methods Appl. Mech. Engrg., 188:713-726, 2000.

[12] H. A. Eschenauer, V. V. Kobelev, and A. Schumacher. Bubble method for topology ans shape optimization of structures. Structural Optimization, 8(1):42-51, 1994.

[13] S. Garreau, P. Guillaume, and M. Masmoudi. The topological asymptotic for pde systems : The elasticity case. SIAM Journal on Control and Optimization, 39(6):1756-1778, 2001.

[14] S. M. Giusti, J. Sokolowski, and J. Stebel. On topological derivatives for contact problems in elasticity. J Optim Theory Appl, 165:279-294, 2015.

[15] P. Guillaume and K. Sid Idris. The topological asymptotic expansion for the dirichlet problem. SIAM Journal on Control and Optimization, 41(4):1042-1072, 2002. 
[16] J. Hadamard. Mémoire sur le problème d'analyse relatif à l'équilibre des plaques élastiques encastrées. Bull. Soc. Math., France, 1907.

[17] F. Hecht. New development in FreeFem++. J. Numer. Math., 20(3-4):251-265, 2012.

[18] A. A. Novotny and J. Sokolowski. Topological derivatives in shape optimization. Springer, Heidelberg, 2013.

[19] S. J. Osher and R. Fedkiw. Level set methods and dynamic implicit surfaces, volume 153. Springer Science \& Business Media, Applied Mathematical Sciences, 2006.

[20] S. J. Osher and J. A. Sethian. Fronts propagating with curvature-dependent speed: Algorithms based on Hamilton-Jacobi formulations. J. Comput. Phys., 79:12-49, 1988.

[21] O. Pironneau. Optimal shape design for elliptic systems. Springer-Verlag, New-York, 1984.

[22] L. Rakotondrainibe. Optimisation topologique des liaisons dans les systèmes mécaniques. PhD thesis, Ecole Polytechnique, 2020. https://tel.archives-ouvertes.fr/tel-03106332.

[23] L. Rakotondrainibe, G. Allaire, and P. Orval. Topology optimization of connections in mechanical systems. Structural and Multidisciplinary Optimization, 61:2253-2269, 2020.

[24] L. Rakotondrainibe, J. Desai, P. Orval, and G. Allaire. Coupled topology optimization of structure and connections in bolted mechanical systems. in preparation, 2021.

[25] B. Samet, S. Amstutz, and M. Masmoudi. The topological asymptotic for the helmholtz equation. SIAM J. Control Optim., 42(5):1523-1544, 2003.

[26] A. Schumacher. Topologieoptimierung von Bauteilstrukturen unter Verwendung von Lochpositionierungskriterien. PhD thesis, UniversitGesamthochschule-Siegen, 1995.

[27] J. A. Sethian. Level set methods and fast marching methods: evolving interfaces in computational geometry, fluid mechanics, computer vision, and materials science, volume 3. Cambridge University Press, 1999.

[28] J. Sokolowski and A. Zochowski. On the topological derivative in shape optimization. SIAM Journal on Control and Optimization, 37:1251-1272, 1999.

[29] J. Sokolowski and A. Zochowski. Topological derivatives of shape functionals for elasticity systems. Mechanics of Structures and Machines, 29:333-351, 2001.

[30] J. Sokolowski and J. P. Zolesio. Introduction to shape optimization. Springer-Verlag, Berlin, 1992.

[31] G. G. Stokes. On the effect of the internal friction of fluids on the motion of pendulums. Trans. Camb. Phil. Soc., 9(II):8-106, 1851. 